\title{
Global and regional evaluation of energy for water
}

4

5 Yaling Liu ${ }^{1, *}$, Mohamad Hejazi ${ }^{1}$, Page Kyle ${ }^{1}$, Son H. Kim ${ }^{1}$, Evan Davies ${ }^{2}$, Diego G.

6 Miralles $^{3,4}$, Adriaan J. Teuling ${ }^{5}$, Yujie $\mathrm{He}^{6}$, Dev Niyogi ${ }^{7}$

$7 \quad{ }^{1}$ Pacific Northwest National Laboratory, Joint Global Change Research Institute, 5825

8 University Research Court, College Park, Maryland, 20740, USA

$9{ }^{2}$ Department of Civil and Environmental Engineering, University of Alberta, Alberta, T6G 1H9,

10 Canada

$11{ }^{3}$ Department of Earth Sciences, VU University, Amsterdam, $1081 \mathrm{HV}$, The Netherlands

$12{ }^{4}$ Laboratory of Hydrology and Water Management, Ghent University, Ghent, B-9000, Belgium

$13{ }^{5}$ Hydrology and Quantitative Water Management Group, Wageningen University, Wageningen,

14 6708PB, The Netherlands

$15{ }^{6}$ Department of Earth System Science, University of California, Irvine, California, 92697, USA

$16{ }^{7}$ Department of Agronomy and Department of Earth, Atmospheric and Planetary Sciences,

17 Purdue University, West Lafayette, Indiana, 47907, USA

18 * Corresponding author. Phone: +1-765-775-6055; Fax: +1-301-314-6719; E-mail address:

19 yaling.liu@pnnl.gov 
20 The supporting information describes the detailed methodology and adjustment to energy

21 intensity and water withdrawal, and also provides details of the uncertainty analysis. It has 4

22 figures, 9 tables and 1 appendix, with 38 double-spaced pages.

\section{Water data processing}

Given that many of the combinations of process, country, and time period in FAO

26 AQUASTAT are missing values, the first step to estimating E4W is to replace missing values

27 with approximations. Several techniques, including inverse distance weighting, ${ }^{1}$ nearest

28 neighbour, linear interpolation/extroplation based on associated variables, are used to fill gaps in the FAO AQUASTAT water use dataset (2). The filling is based on the number $\left(\mathrm{N}_{\text {avail }}\right)$ and value $\left(\mathrm{V}_{\text {avail }}\right)$ of available data points among eight 5-year periods between 1973-1977 and 2008-2012, as well as the nature of the different variables. AQUASTAT estimates in the USA are replaced

32 with data from USGS inventories, ${ }^{3-10}$ which provide more details with fewer missing values or 33 apparent inter-annual discrepancies. The data availability of key variables varies from time to 34 time, with a peak at 2000 (Fig. S1). National totals of water withdrawals are interpolated or 35 extrapolated, and subsequently assigned to specific components whose shares are computed as described below. Water use by individual components in multi-country regions is then estimated

37 by adding the corresponding values from the constituent countries. specific country (200 in total), there are several alternative approaches for data filling: 
42 that of the first available data point, those between were estimated by inverse distance weighting 43 method (1), and those after were extrapolated by following the temporal linear trend of the 44 nearest two data points.

45 2) If $N_{\text {avail }}=1$, the missing data are estimated by multiplying the $V_{\text {avail }}$ with the 46 population ratio between the period of missing data that corresponds to the $\mathrm{V}_{\text {avail. }}$.

47 3) If $\mathrm{N}_{\text {avail }}=0$, the missing data for the country are calculated by multiplying its 48 population with the average water use per capita of countries within the specific region for which 49 data are available.

50 While the method described above is generally used for filling missing values, proxies 51 other than population are used for several water use variables. We use irrigated land area by 52 nation and over time ${ }^{11}$ in order to interpolate or extrapolate irrigation water withdrawals.

53 Similarly, for industrial water withdrawals, we use industrial value added, ${ }^{12-13}$ and for municipal 54 water withdrawals, we use the estimated population with access to improved water sources. ${ }^{14-15}$ 55 For desalinated water production, a different method was used than 56 interpolation/extrapolation because many countries did not operate desalination plants, 57 particularly in the early years of the dataset. In this dataset, missing values were simply set to 58 zero, with the exception of missing values in countries and years where the same country had 59 reported values in prior years. In this case, then the values for the missing years were assumed to 60 be the same as the last earlier period with reported values. To determine the agricultural, industrial and municipal shares of the TWW, and the fresh 62 surface and groundwater withdrawal shares of the TFWW, the following approach was 
employed, based on the number $\left(\mathrm{N}_{\text {avail_share }}\right)$ and value $\left(\mathrm{V}_{\text {avail_share }}\right)$ of share that could be explicitly computed from available data among the eight 5-year periods during 1973-2012:

1) If $\mathrm{N}_{\text {avail_share }}>=1$, the missing share was filled with the $\mathrm{V}_{\text {avail_share }}$ from the nearest time period.

2) If $\mathrm{N}_{\text {avail_share }}=0$, the missing share was filled with region-average share.

Next, the TWW and TFWW were multiplied by the derived water withdrawal shares computed for each sector and water source. The difference between the TWW and TFWW was the recycled water use - in other words, the sum of direct use of treated municipal wastewater, agricultural drainage water and desalinated water. The derivation of activity-specific shares between surface water and groundwater for water activities in each sector is described in Section S2, below.

Note that AQUASTAT does not report seawater withdrawal for power plant cooling, although this may be a significant quantity of water in regions such as Japan, the Middle East, the USA, and Korea, all of which operate a number of large, coastal thermoelectric power plants that use once-through flow cooling systems. To estimate this water quantity, we use data from Kim et al. (2015) ${ }^{16}$ and Davies et al. $(2013)^{20}$ to develop a time-series of seawater withdrawals. Additionally, several scalars are used to account for the portion of water that actually involves energy consumption in our evaluation of E4W. A scalar involved in irrigation is the percentage of the irrigation area that is equipped for power irrigation, defined as the area where electric pumps are used for water supply from the source to the scheme. ${ }^{1}$ A second scalar that pertains to surface industrial water use is the percent of industrial water used for thermoelectric power plant cooling. ${ }^{3}$ Readers are referred to the Section S2, below, for details. 
Our data-filling approaches may have limitations. Population-based extrapolations

86 disregard the effects of technological change. Additionally, municipal water purchased by

87 industrial consumers is assigned to the municipal sector; while this does not bias the total base-

88 year estimates of $\mathrm{E} 4 \mathrm{~W}$, it does increase the municipal share relative to the industrial share, and

89 consequently will bias sectoral energy demand projections. Finally, using the area shares of

90 power irrigation as an approximation of the volume shares of power irrigation may also induce

91 bias, because of the omission of spatial variability in irrigation intensity.

\section{2. Scalars and adjustment for water use}

93 Two scalars including the percent of irrigation area that is equipped for power irrigation

$94(\alpha 1)$, and the percent of industrial water used for thermoelectric power plant cooling $(\alpha 2)$ are

95 applied to agricultural and industrial water use, respectively, in the assessment of energy for

96 water $(\mathrm{E} 4 \mathrm{~W})$. The same approach as described in Section S1 is used to fill any gaps in the raw

97 data for the two scalars. When applying $\alpha 1$, we collect information for the share of groundwater

98 in irrigation withdrawal (gw_irr_shr) from different sources, including literature and FAO

99 reports $(1,17-18)$, and there are two cases:

100

1) If $\alpha 1>=$ gw_irr_shr, we assume all groundwater used for irrigation is using power and

101 the remaining portion comes out of surface water.

2) If $\alpha 1<$ gw_irr_shr, we assume only a portion of $\alpha 1$ groundwater irrigation requires

103 power, and then the remaining groundwater irrigation and surface water irrigation is gravity-

104 driven. 
The $\alpha 2$ is derived from region-specific thermoelectric water use $\mathrm{e}^{3}$ and total industrial

106

107

108

109

110

111

112 122 is denoted as gw_irr):

$123 \quad \frac{\text { groundwater }-g w_{-} \text {irr }}{\text { TWW-irrigation }}=\frac{0.42-0.3825}{4.205-3.318}=4.23 \%$ seawater withdrawal for cooling is listed in Table S6.

$$
\frac{T W W-\text { irrigation }}{T .205-3.318}=4.23 \%
$$

water use, where we assume all water used for thermoelectric power plant cooling is from surface water as the portion from groundwater is small. ${ }^{10}$ The time-evolving and region-specific $\alpha 2$ is listed in Table S5. We apply an EI of $0.073 \mathrm{kWh} / \mathrm{m}^{3}$ (ref. 19) to the process of source and conveyance for the cooling water, and assume that no other E4W related processes apply to this water (i.e., no treatment, distribution, or post-use wastewater treatment is needed). Seawater used for power plant cooling is also included in the E4W evaluation of this process; estimated

The following example illustrates how the water balances are closed for Zimbabwe with the following characteristics. The total water withdrawal for Zimbabwe in 2002 is 4.205 bcm, and the shares between surface and groundwater use are $90 \%$ by $10 \%$ (3.785 and $0.42 \mathrm{bcm})$. The shares between agricultural, industrial and municipal are $78.9 \%, 7.1 \%$ and $14 \%(3.318,0.298$ and $0.589 \mathrm{bcm}$ ), respectively. In addition, irrigation accounts for $100 \%$ of agricultural water use $(3.318 \mathrm{bcm})$, and $11.53 \%$ of irrigation uses groundwater $(0.3825 \mathrm{bcm}$, here assume the area share of groundwater irrigation as an approximation of the volume share). Thus, the groundwater share for water use activities other than irrigation - other agricultural (i.e., livestock and aquaculture) and industrial and municipal water use - is calculated as $4.23 \%$ as below (groundwater irrigation

For further derivation of surface/groundwater share for each activity within the other water use sectors (e.g., manufacturing), similar methods are applied. 


\section{Energy intensity survey and adjustment}

Due to real-world variability in water operations systems that lead to differences in average energy efficiency, there is no single set of energy intensity (EI) assumptions for each E4W process that is likely provide a good approximation of the actual energy intensity of each process in all regions and over time. Moreover, using the geographic and temporal information of specific studies in order to estimate region- and time-specific EI values is also problematic, due to differences in data gathering techniques and terminology.

To deal with this source of uncertainty, we use three levels of EI for each process that correspond to different percentiles of reported values in the literature, as described below. Upon extensive literature survey (see Table S2a, S2b and S3), ${ }^{19,21-96}$ we sample five data points for each individual reported range (single data point for single reported EI) by using uniform distribution for each process in each individual literature, and present the minimum, maximum, and the $25^{\text {th }}, 50^{\text {th }}$ and $75^{\text {th }}$ percentile, where the $75^{\text {th }}$ percentile represents the upper level of EI reported in literature.

While default EI values are used for most processes, regions, and time periods, efforts are made to capture spatial and temporal variations in EI based on available information.

Desalination in particular has seen significant reduction in EI for the reverse osmosis (RO) technologies, ${ }^{80}$ which are captured in our evaluation (Table S3). Region-specific mean groundwater depths derived from Fan et al. (2013) ${ }^{97}$ are used to linearly scale the EI for groundwater pumping. Additionally, information on EI for specific countries in individual studies (see Table S2a, S2b, and S3) is also used to adjust the EI assumptions, especially municipal water supply. ${ }^{29,95}$ Note that the process of water distribution is not applicable to 
148 industrial water, as industrial water use in AQUASTAT only refers to self-supplied industries not 149 connected to the public distribution network. ${ }^{1}$ The EI scaling methods are detailed below. Country-specific mean groundwater depths (GWD) are obtained from Fan et al. (2013) ${ }^{97}$

151 and then aggregated to region-specific GWD. Then the EI values for groundwater pumping for

152 other regions are estimated as EPRI $(2002)^{19}$ EI values, multiplied by the ratio of a region-

153 specific GWD over that of the USA. While the majority of the literature on municipal water 154 supply is for USA (Table S2a, S2b), variations have been revealed across different countries by 155 Liu et al. $(2012)^{29}$ - for example, the ratios of EI between the reported countries (i.e., Russia, 156 China, Brazil and India) and USA ${ }^{19}$ are around 1.5, 1.2, 2 and 1.6, respectively. These ratios are 157 then multiplied by the $25^{\text {th }}, 50^{\text {th }}$ and $75^{\text {th }}$ percentile EIs of each process for municipal water 158 supply derived from the literature survey (Table S1, except desalination), for these four regions. 159 The EI after this scaling is still within the EI range as reported by literature in all cases (Fig. 1, 160 Table S2a, S2b). Note that the EI range reported in Liu et al. (2012) ${ }^{29}$ was not included in Fig. 1 161 as the study only addresses total water supply EI, not the individual water processes reported in 162 Fig. 1.

\section{Estimation of conversion ratio from primary energy to electricity}

The vast majority of energy consumed by water-related processes is in the form of

165 electricity, as this tends to be the fuel used to pump, pressurize and treat water. In order to 166 provide a more complete assessment of the relative importance of water-related processes in 167 each region's energy system, the electricity used by these processes is translated to total primary 168 energy prior to adding to any direct primary (thermal) energy use by the water-related processes.

169 To perform this calculation, we first add up all primary energy used to generate electricity in 
170 each region and in each time period, according to the IEA Energy Balances. ${ }^{86}$ Consistent with 171 the accounting conventions of the IEA, the primary energy input to hydropower and other

172 renewables is set equal to the electricity output, and the primary energy input to nuclear is equal

173 to three times the electricity output. This is divided by the total electricity consumption - i.e.,

174 delivered to all end users - in the given region and time period. In this fashion, the calculated

175 conversion ratio (CR) accounts for energy losses in both (a) transformation from primary energy

176 to electricity and (b) electricity transmission and distribution. Specifically, the time-evolving and

177 region-specific CR is calculated as follows:

178

$$
C R_{i}=\frac{\sum_{j=1}^{n} \sum_{p=1}^{m} P E_{i, j, p}}{\sum_{j=1}^{n} E L E C_{i, j}}
$$

179 where $i, j$ and $p$ stand for the $i$-th time period, the $j$-th country within the specific geopolitical

180 region and the $p$-th fuel type, $C R$ is the region-specific conversion ratio from electricity to

181 primary energy, $P E$ stands from primary energy input used to generate electricity and $E L E C$

182 represents the delivered electricity. The time-evolving and region-specific $C R$ values are listed in 183 Table S5.

\section{Uncertainty analysis}

185 Non-parametric bootstrapping approach is utilized here to investigate the uncertainties of E4W 186 estimates derived from the 35 parameters involved - 34 EI parameters illustrated in Fig. 1 and 1

187 CR parameter. First, we sample a size of 1000 (with replacement) for each parameter from their 188 original sample, and thus we have 1000 combinations of the 35 parameters (without permutation). 189 Second, we calculate the E4W by using the 1000 combinations of parameters. Third, 1000 
190 bootstraps were performed. Fourth, we compute the statistics for the E4W estimates derived 191 from the bootstrapping method, so as to quantify the uncertainty of E4W - 95\% confidence 192 interval for the E4W and the share of E4W in total primary energy consumption (Fig. 6).

1946 Downloadable data for country-level key variables and estimated E4W for 2010

195 To make this work more useful to the community, we provide country-level information of 196 E4Win an Excel file (i.e., the supplementary Excel file Appendix S1). The Excel file has two 197 sheets, the one named "data" provides the information of country, water withdrawal, power 198 irrigation, share between surface water and groundwater for irrigation and other water-use 199 activities, variables for estimating process-level water-use amount, adjusting coefficient for EI, 200 and E4W estimates at water sources, water-use processes and end-use sectors levels. The other 201 sheet named "variables" describes the denotations of each variable in the "data" sheet and the 202 corresponding unit. 


\section{Figure Captions}

204 Figure S1 Coverage of available data for the key variables in AQUASTAT from 1973 to 2012, 205 calculated as the ratio of sum of available raw data to the corresponding estimated global total 206 for a specific variable in a specific time.

207 Figure S2 Global map of the 14 geopolitical regions used in this study, which is defined by the 208 Global Change Assessment Model (GCAM, ref 99).

209 Figure S3 Variations of energy for water (E4W) for desalination by technologies and regions 210 during 1973-2012. The label names for the upper panel are composed in the format of water

211 source-technology $(\mathrm{sw}=$ seawater, $\mathrm{br}=$ brackish water, $\mathrm{MSF}=$ multistage flash distillation, MED

$212=$ multi-effect distillation, $\mathrm{RO}=$ reverse osmosis, $\mathrm{ED}=$ electrodialysis $).$

213 Figure S4 Country-scale analysis of energy for water (E4W) in 2010: a) E4W per capita (10 ${ }^{-9}$

214 EJ); and b) share of E4W in total primary energy consumption (TPEC) (\%). The maps are 215 generated via ESRI ArcMap10.2, and the results presented are from analysis in this study and the 216 underlying country boundaries are from ESRI World Countries dataset. 


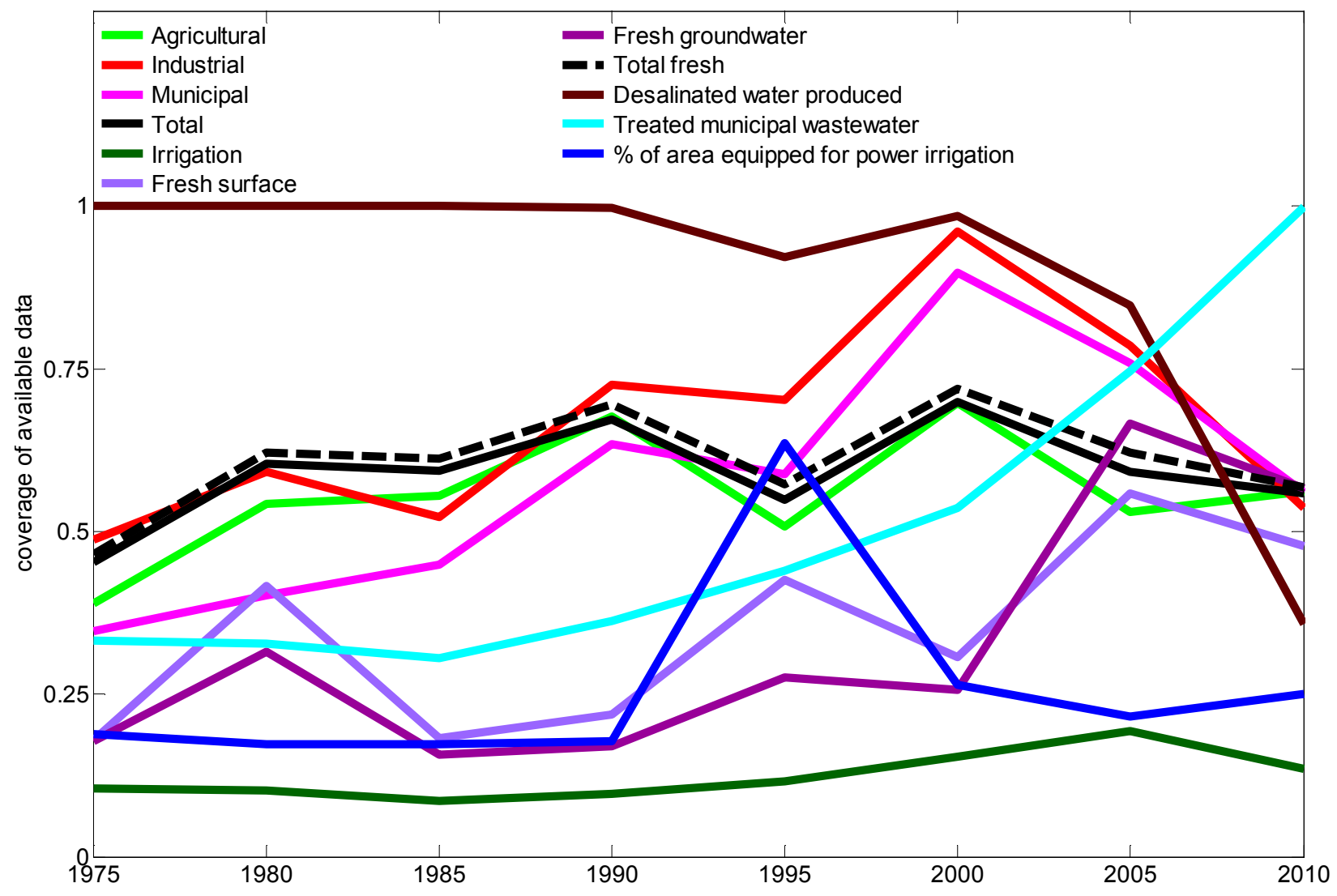


Figure S2

221

222 


\section{Figure S3}
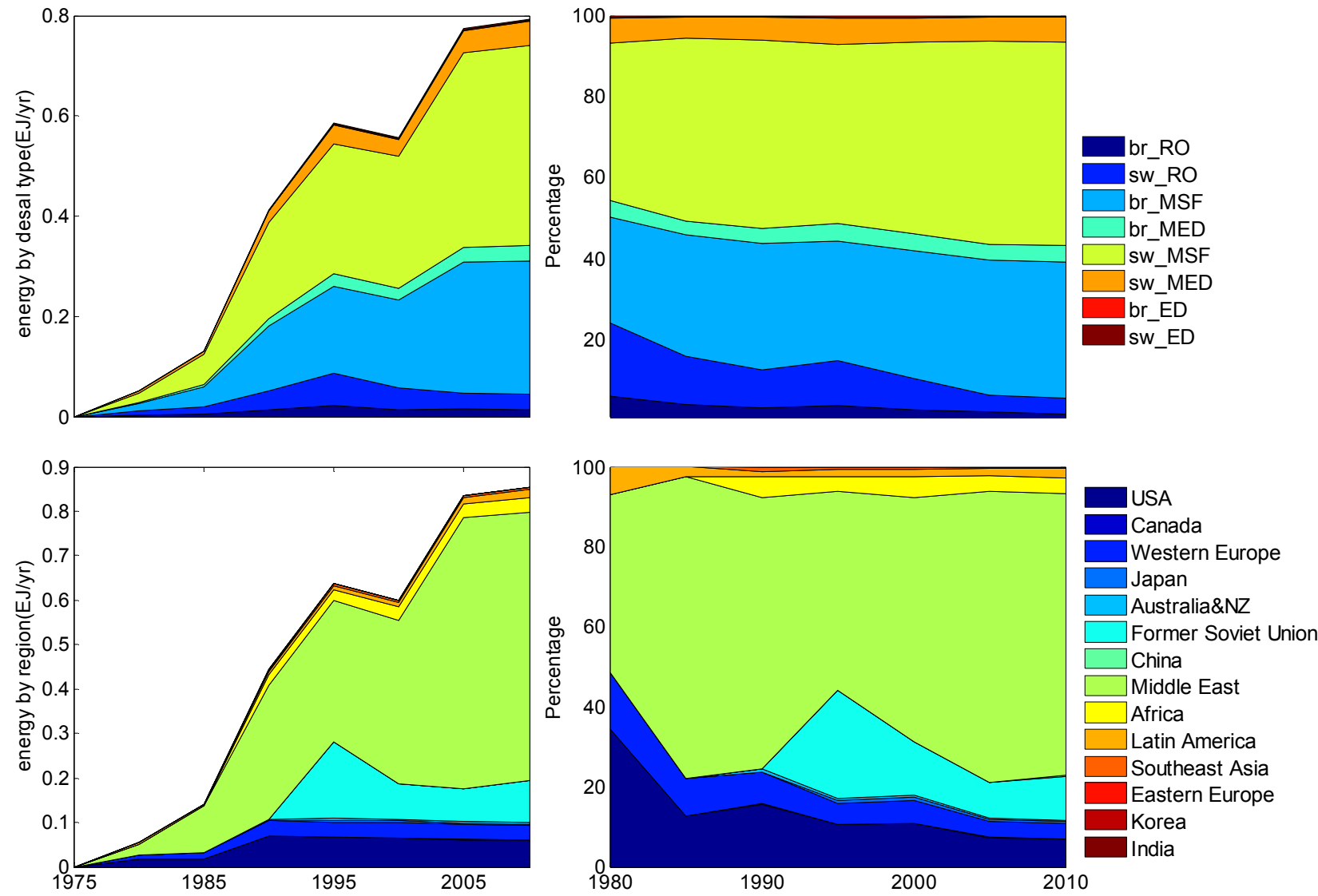
(a)

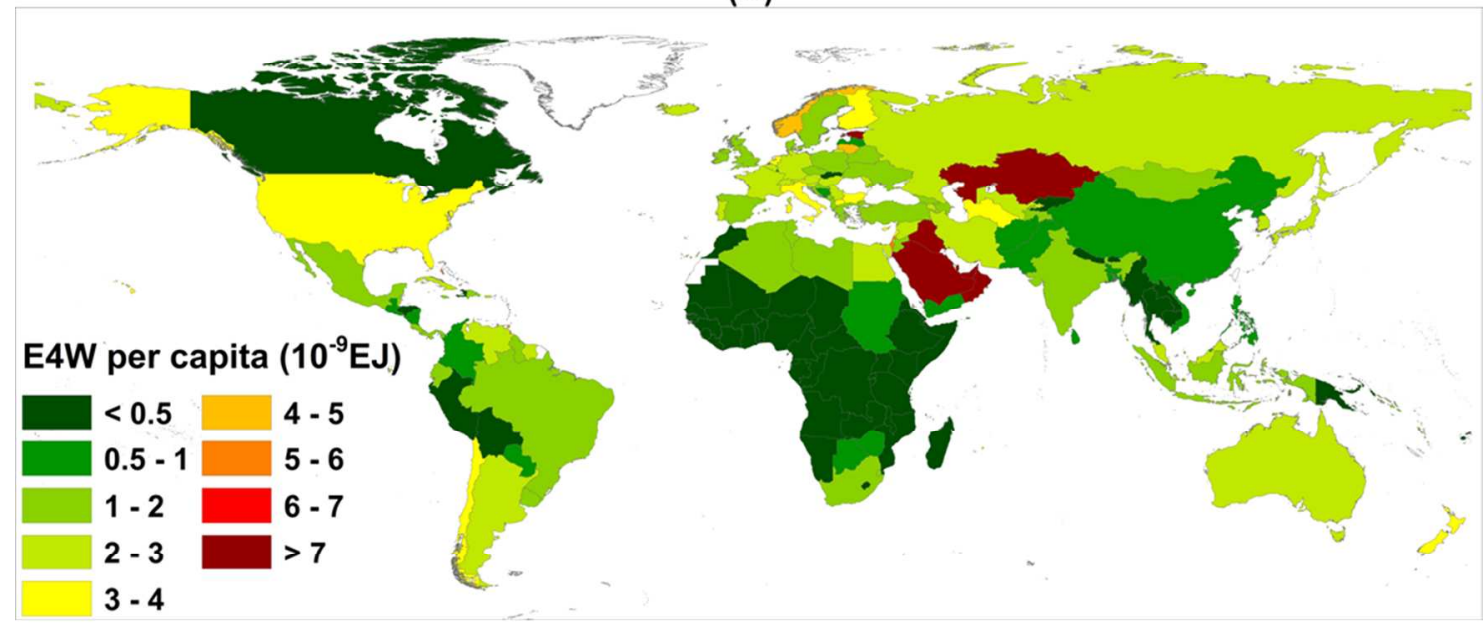

(b)

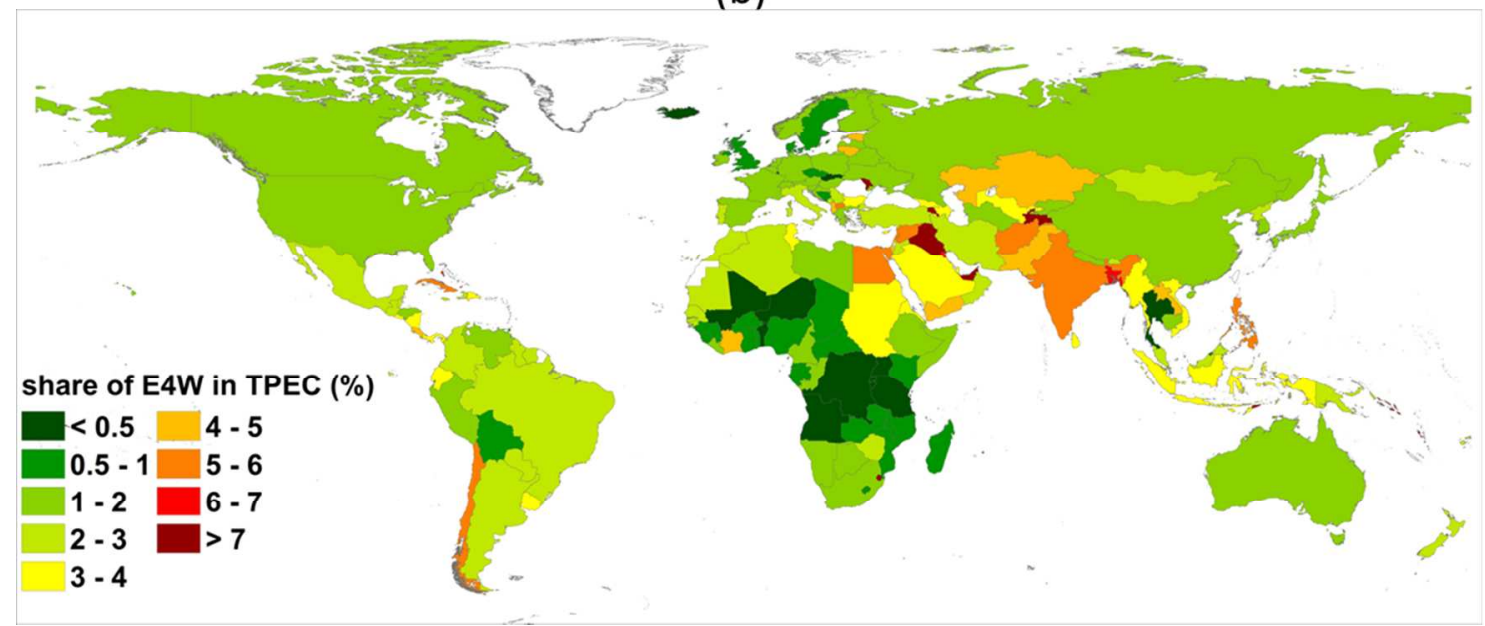


229 Table S1 Energy intensity $\left(\mathrm{kWh} / \mathrm{m}^{3}\right)$ for different processes across different sectors and sources

$230(\mathrm{sf}=$ surface water, $\mathrm{gw}=$ groundwater, $\mathrm{ww}=$ wastewater, $\mathrm{sw}=$ seawater, $\mathrm{br}=$ brackish water,

$231 \mathrm{MSF}=$ multistage flash distillation, $\mathrm{MED}=$ multi-effect distillation, $\mathrm{RO}=$ reverse osmosis, $\mathrm{ED}=$

232 electrodialysis).

\begin{tabular}{|c|c|c|c|c|c|c|}
\hline & water process & sector/technology & source & $25 \%$ EI & $50 \%$ EI & $75 \%$ EI \\
\hline \multirow{20}{*}{ 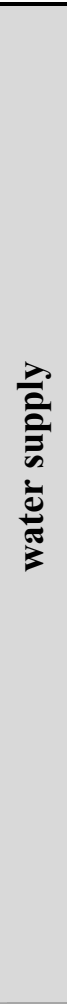 } & \multirow{6}{*}{ source/conveyance } & \multirow{2}{*}{ Agriculture } & sf & 0.050 & 0.079 & 0.100 \\
\hline & & & gw & 0.100 & 0.185 & 0.300 \\
\hline & & \multirow[t]{2}{*}{ Industrial } & sf & 0.050 & 0.079 & 0.100 \\
\hline & & & gw & 0.100 & 0.185 & 0.300 \\
\hline & & \multirow[t]{2}{*}{ Municipal } & sf & 0.050 & 0.079 & 0.100 \\
\hline & & & gw & 0.100 & 0.185 & 0.300 \\
\hline & \multirow[t]{4}{*}{ water treatment } & \multirow[t]{2}{*}{ Industrial } & sf & 0.057 & 0.160 & 0.300 \\
\hline & & & gw & 0.102 & 0.195 & 0.340 \\
\hline & & \multirow[t]{2}{*}{ Municipal } & sf & 0.120 & 0.220 & 0.400 \\
\hline & & & gw & 0.165 & 0.250 & 0.400 \\
\hline & \multirow[t]{4}{*}{ water distribution } & \multirow[t]{2}{*}{ Industrial } & sf & 0 & 0 & 0 \\
\hline & & & gw & 0 & 0 & 0 \\
\hline & & \multirow[t]{2}{*}{ Municipal } & sf & 0.170 & 0.247 & 0.339 \\
\hline & & & gw & 0.170 & 0.247 & 0.339 \\
\hline & \multirow[t]{6}{*}{ desalination } & MSF & $\mathrm{sw} / \mathrm{br}$ & 22 & 62.56 & 83 \\
\hline & & MED & $\mathrm{sw} / \mathrm{br}$ & 29 & 54.25 & 72.65 \\
\hline & & $\mathrm{RO}$ & SW & 3.3 & 4.75 & 6.5 \\
\hline & & & br & 1 & 1.5 & 2.5 \\
\hline & & ED & sf & 1.8 & 2.5 & 4 \\
\hline & & & br & 1.24 & 2.4 & 3.39 \\
\hline \multirow{6}{*}{ 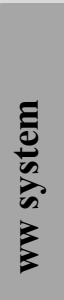 } & \multirow[t]{2}{*}{ ww collection } & industrial & $\mathrm{sf} / \mathrm{gw}$ & 0.037 & 0.067 & 0.175 \\
\hline & & municipal & $\mathrm{sf} / \mathrm{gw}$ & 0.039 & 0.058 & 0.157 \\
\hline & \multirow[t]{2}{*}{ ww treatment } & industrial & $\mathrm{sf} / \mathrm{gw}$ & 0.328 & 0.650 & 0.870 \\
\hline & & municipal & $\mathrm{sf} / \mathrm{gw}$ & 0.300 & 0.479 & 0.752 \\
\hline & \multirow[t]{2}{*}{ ww discharge } & industrial & sf/gw & 0.020 & 0.060 & 0.100 \\
\hline & & municipal & $\mathrm{sf} / \mathrm{gw}$ & 0.020 & 0.060 & 0.100 \\
\hline
\end{tabular}


Table S2a. Summary of energy intensity values $\left(\mathrm{kWh} / \mathrm{m}^{3}\right)$ for water supply system by water sources and end use sectors $(\mathrm{sf}=$ surface water, gw $=$ groundwater, $\mathrm{ww}=$ wastewater, ag = agricultural, ind = industrial, mun = municipal, $\mathrm{NS}=$ not specified, $\mathrm{L}=\mathrm{low}, \mathrm{H}=\mathrm{high}$ ).

\begin{tabular}{|c|c|c|c|c|c|c|c|c|c|c|c|c|c|c|c|c|c|c|c|c|c|}
\hline & \multicolumn{4}{|c|}{ Aggregated supply } & \multicolumn{3}{|c|}{ source water abstraction } & \multicolumn{5}{|c|}{ conveyance } & \multicolumn{4}{|c|}{ water treatment } & \multicolumn{4}{|c|}{ water distribution } & \multirow[t]{2}{*}{ Study region } \\
\hline & \multirow{2}{*}{$\begin{array}{ll}\mathrm{NS} \\
\mathrm{NS} \\
\end{array}$} & \multirow{2}{*}{$\begin{array}{l}\mathrm{ag} \\
\mathrm{sf} / \mathrm{gw} \\
\end{array}$} & \multirow{2}{*}{$\begin{array}{l}\text { ind } \\
\text { sf/gw } \\
\end{array}$} & \multirow{2}{*}{$\begin{array}{l}\text { mun } \\
\text { sf/gw } \\
\end{array}$} & \multirow{2}{*}{$\begin{array}{ll}\mathrm{NS} \\
\mathrm{NS} \\
\end{array}$} & \multicolumn{2}{|c|}{ mun } & \multicolumn{3}{|c|}{$\mathrm{NS}$} & \multirow{2}{*}{$\begin{array}{ll}\mathrm{ag} \\
\mathrm{NS} \\
\end{array}$} & \multirow{2}{*}{$\begin{array}{l}\text { mun } \\
\text { NS } \\
\end{array}$} & \multicolumn{3}{|c|}{$\mathrm{NS}$} & \multirow{2}{*}{$\frac{\mathrm{mun}}{\mathrm{NS}}$} & \multirow{2}{*}{$\begin{array}{ll}\mathrm{NS} \\
\mathrm{NS} \\
\end{array}$} & \multicolumn{3}{|c|}{ mun } & \\
\hline & & & & & & sf & $\mathrm{gw}$ & NS & sf & gw & & & NS & sf & gw & & & $\mathrm{NS}$ & sf & $\mathrm{gw}$ & \\
\hline $\begin{array}{l}\text { Burt et al. } 2011 \\
\text { Burton } 1996\end{array}$ & & & & & & 0.03 & 0.16 & & & $0-0.75^{\mathrm{b}}$ & & & & 0.04 & .002 & & & & 0.32 & 0.32 & $\begin{array}{l}\text { California, } \\
\text { USA } \\
\text { USA }\end{array}$ \\
\hline $\begin{array}{l}\text { Carlson and } \\
\text { Wallburger } 2007\end{array}$ & & & & & $\begin{array}{l}0.07- \\
0.79\end{array}$ & & & & & & & & & & & $\begin{array}{l}0.07- \\
0.53\end{array}$ & $\begin{array}{l}0.07- \\
0.46\end{array}$ & & & & USA \\
\hline CEC 2005 & & & & & & & & $0-3.7^{\mathrm{b}}$ & & & & & & & & & & & $\begin{array}{l}0.19- \\
0.32\end{array}$ & & $\begin{array}{l}\text { California, } \\
\text { USA }\end{array}$ \\
\hline CEC 2006 & & & & & & & & $\begin{array}{l}0.55- \\
2.58^{\mathrm{b}}\end{array}$ & & & & & & & & 0.03 & & & $\begin{array}{l}0.19- \\
0.32\end{array}$ & & $\begin{array}{l}\text { California, } \\
\text { USA }\end{array}$ \\
\hline Cheng et al. 2002 & & & & & & & & & & & & & & & & 0.22 & & 0.17 & & & Taiwan \\
\hline Cohen et al. 2004 & & $0.28^{\mathrm{a}}$ & & & $0.54^{\mathrm{be}}$ & & $\begin{array}{l}0.14- \\
0.6\end{array}$ & 2.39 & $2.43^{\mathrm{b}}$ & $\begin{array}{l}0.14- \\
0.53^{\mathrm{b}}\end{array}$ & & & $\begin{array}{l}0.03- \\
0.06\end{array}$ & & & & $0.27^{\mathrm{e}}$ & $\begin{array}{l}0.13- \\
0.76\end{array}$ & & & $\begin{array}{l}\text { California, } \\
\text { USA }\end{array}$ \\
\hline Costa et al. 2006 & & & & & & & & & & & & $0.54^{\mathrm{g}}$ & & & & & & & & & Spain \\
\hline \multirow[t]{8}{*}{ Dhakal et al. 2015} & & & & $\begin{array}{l}0.09- \\
1.92^{\mathrm{a}}\end{array}$ & & & & & & & & & & & & & & & & & Australia \\
\hline & & & & & & & & & & & & $\begin{array}{l}0- \\
1.06\end{array}$ & & & & & & $\begin{array}{l}0.18- \\
0.32\end{array}$ & & & USA \\
\hline & & $0.28^{\mathrm{a}}$ & & & & & & & & & & & & & & & & & & & Canada \\
\hline & & & & & & & & & & & & $\begin{array}{l}0.12- \\
1.13^{\mathrm{g}}\end{array}$ & & & & & & $\begin{array}{l}0.03- \\
0.83\end{array}$ & & & Germany \\
\hline & & & & & & & & & & & & 0.1 & & & & 0.22 & & 0.24 & & & Singapore \\
\hline & & & & & & 0.01 & & & & & & $0.05^{\mathrm{g}}$ & & & & & & 0.08 & & & $\begin{array}{l}\text { Bangkok, } \\
\text { Thailand }\end{array}$ \\
\hline & & & & & 0.3 & & & & & & & $0.17^{\mathrm{g}}$ & & & & & & 0.5 & & & Delhi, India \\
\hline & & & & & $\begin{array}{l}0.15- \\
0.32\end{array}$ & & & & & & & $0.29^{\mathrm{g}}$ & & & & & & 0.13 & & & Tokyo, Japan \\
\hline Elliott et al. 2003 & & & & $\begin{array}{l}0.37- \\
0.45^{\mathrm{a}}\end{array}$ & & & & & & & & & & & & & & & & & $\begin{array}{l}\text { Wisconsin, } \\
\text { USA }\end{array}$ \\
\hline EPA 2008 & & & & & & & & & & & & & & & & & $0.4^{\mathrm{f}}$ & & & & $\begin{array}{l}\text { Massachusetts, } \\
\text { USA }\end{array}$ \\
\hline EPRI 2002 & & $\begin{array}{l}0.08- \\
0.19^{\mathrm{a}}\end{array}$ & $\begin{array}{l}0.08- \\
0.2^{\mathrm{a}}\end{array}$ & $\begin{array}{l}0.37- \\
0.48^{\mathrm{a}}\end{array}$ & & 0.03 & 0.16 & & & & & & & $\begin{array}{l}0.02- \\
0.03\end{array}$ & & & & & 0.32 & & USA \\
\hline EPRI 2013 & $\begin{array}{l}0.5- \\
0.86^{\mathrm{a}}\end{array}$ & & & $\begin{array}{l}0.42- \\
0.56^{\mathrm{a}}\end{array}$ & & $\begin{array}{l}0.03- \\
0.05\end{array}$ & $\begin{array}{l}0.2- \\
0.32\end{array}$ & & & & & & & & & $\begin{array}{l}0.07- \\
0.38\end{array}$ & $\begin{array}{l}0.21- \\
0.30\end{array}$ & & $\begin{array}{l}0.21- \\
0.36\end{array}$ & $\begin{array}{l}0.21- \\
0.36\end{array}$ & USA \\
\hline Kneppers et al. 2009 & & & & & & & & & & & & & & $\begin{array}{l}0.04- \\
0.31\end{array}$ & & & & & & & New Zealand \\
\hline
\end{tabular}


Continued Table S2a. Summary of energy intensity values $\left(\mathrm{kWh} / \mathrm{m}^{3}\right)$ for water supply system by water sources and end use sectors $(\mathrm{sf}=$ surface water, $\mathrm{gw}=$ groundwater, $\mathrm{ww}=$ wastewater, $\mathrm{ag}=$ agricultural, $\mathrm{ind}=$ industrial, mun $=$ municipal, $\mathrm{NS}=$ not $\mathrm{specified}, \mathrm{L}=\mathrm{low}, \mathrm{H}=\mathrm{high}$ )

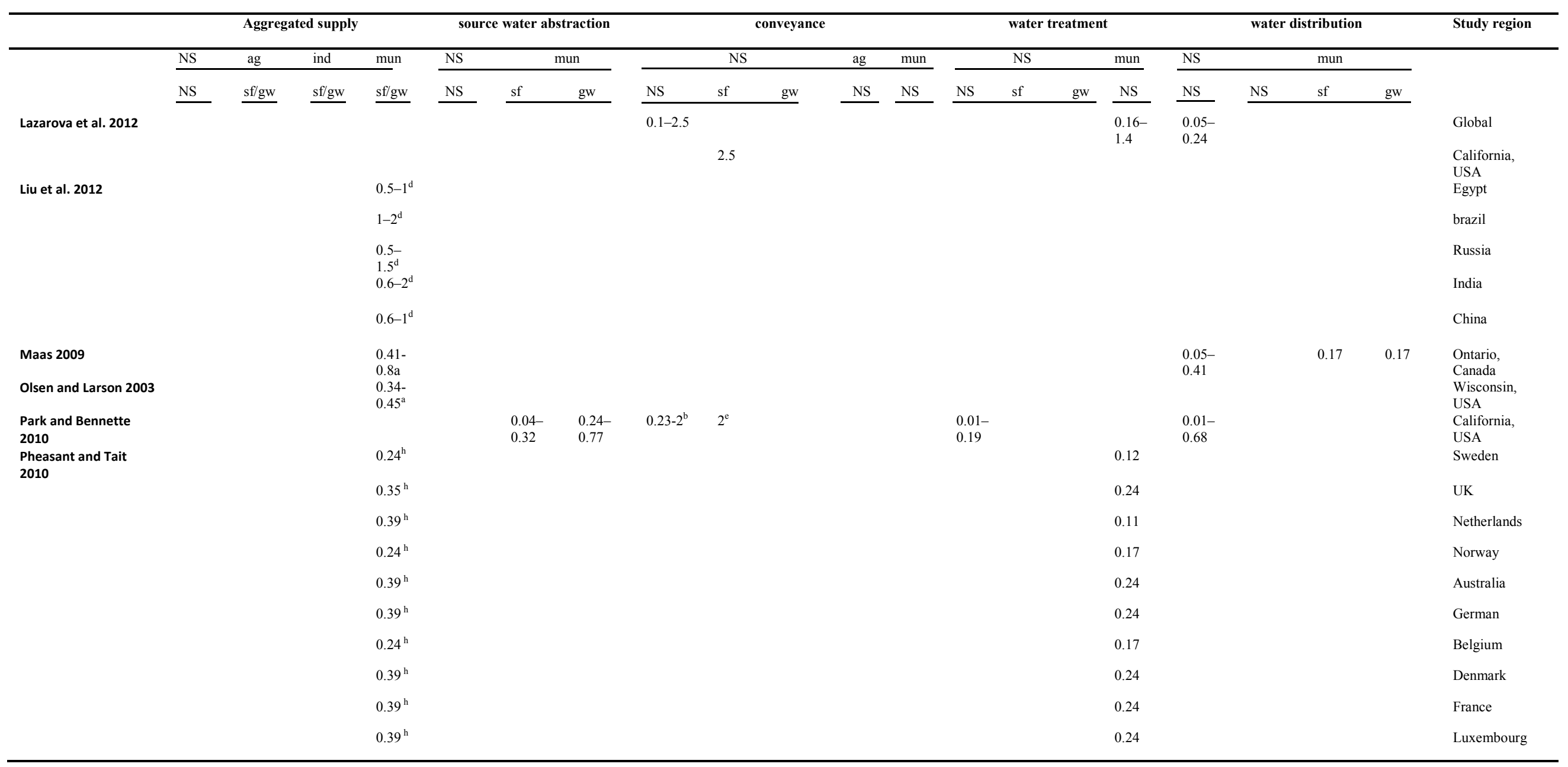


Continued Table S2a. Summary of energy intensity values $\left(\mathrm{kWh} / \mathrm{m}^{3}\right)$ for water supply system by water sources and end use sectors $(\mathrm{sf}=$ surface water, $\mathrm{gw}=$ groundwater, $\mathrm{ww}=$ wastewater, ag = agricultural, ind $=$ industrial, mun $=$ municipal, $\mathrm{NS}=$ not $\mathrm{specified,} \mathrm{L}=$ low, $\mathrm{H}=$ high) .

\begin{tabular}{|c|c|c|c|c|c|c|c|c|c|c|c|c|c|c|c|c|c|c|c|c|c|}
\hline & \multicolumn{4}{|c|}{ Aggregated supply } & \multicolumn{3}{|c|}{ source water abstraction } & \multicolumn{5}{|c|}{ conveyance } & \multicolumn{4}{|c|}{ water treatment } & \multicolumn{4}{|c|}{ water distribution } & \multirow[t]{2}{*}{ Study region } \\
\hline & NS & $\mathrm{ag}$ & ind & mun & NS & & in & & NS & & ag & mun & & NS & & mun & NS & & mun & & \\
\hline & NS & sf $/ \mathrm{gw}$ & sf $/ \mathrm{gw}$ & sf/gw & NS & sf & $\mathrm{gw}$ & NS & sf & gw & NS & NS & NS & sf & gw & NS & NS & NS & sf & gw & \\
\hline \multirow[t]{2}{*}{$\begin{array}{l}\text { Plappally and Lienhard } \\
2012\end{array}$} & & & & & & & & & $0-4^{b}$ & & & & 0.11 & & & & & & & & Spain \\
\hline & & & & & & & & & $\begin{array}{l}1.6- \\
2.6^{\mathrm{b}} \\
0.06- \\
3.3^{\mathrm{b}}\end{array}$ & & & & $\begin{array}{l}0.01- \\
0.2\end{array}$ & & & & & & & & $\begin{array}{l}\text { California, } \\
\text { USA } \\
\text { Australia }\end{array}$ \\
\hline \multirow[t]{4}{*}{$\begin{array}{l}\text { Plappally and Lienhard } \\
2012\end{array}$} & & & & & & & & & & $\begin{array}{l}0.25- \\
3.02^{\mathrm{b}}\end{array}$ & & & $\begin{array}{l}0.38- \\
1.44\end{array}$ & & & & & & & & $\begin{array}{l}\text { Ontario, } \\
\text { Canada }\end{array}$ \\
\hline & & & & & & & & & & & & & $\begin{array}{l}0.16- \\
0.25\end{array}$ & & & & & & & & Taiwan \\
\hline & & & & & & & & $\begin{array}{l}0.4- \\
3.59^{\mathrm{b}}\end{array}$ & & $\begin{array}{l}0.04- \\
0.6\end{array}$ & & & $\begin{array}{l}0.18- \\
0.47\end{array}$ & $\begin{array}{l}0.06- \\
0.16\end{array}$ & $\begin{array}{l}0.15- \\
0.5\end{array}$ & & $\begin{array}{l}0.1- \\
0.41\end{array}$ & & & & USA \\
\hline & & & & & & & & & & & & & $\begin{array}{l}0.15- \\
0.44\end{array}$ & & & & & & & & New Zealand \\
\hline Pirne and Yonkin 2008 & & & & $\begin{array}{l}0.16- \\
0.29^{\mathrm{a}}\end{array}$ & & & & & & & & & & & & & & & & & $\begin{array}{l}\text { New York, } \\
\text { USA }\end{array}$ \\
\hline Raluy et al. 2005 & & & & & & & & & 1.79 & & & & & & & & & & & & Spain \\
\hline $\begin{array}{l}\text { Sauer and Kimber } \\
2003\end{array}$ & & & & & & & & & & & & & & & & & 0.10 & & & & Iowa, USA \\
\hline Siddiqi et al. 2011 & & & & & & 0.1 & 0.4 & & & & 0.4 & $0-3.7$ & & & & & & & & & Compiled \\
\hline WEF 2009 & & & & & & & & & & & & & & $0-0.3$ & & & & & & & USA \\
\hline $\mathbf{L}$ & & & & 0.09 & 0.07 & 0.03 & 0.14 & 0 & 0.06 & 0 & & 0 & 0.01 & 0.02 & 0.002 & 0.03 & 0.01 & 0.03 & 0.17 & 0.17 & \\
\hline H & & & & 1.92 & 2.58 & 0.32 & 0.77 & 3.7 & 3.3 & 3.02 & & 3.7 & 1.44 & 0.31 & 0.5 & 1.4 & 0.68 & 0.76 & 0.36 & 0.36 & \\
\hline
\end{tabular}

a: energy consumption for raw water pumping, conveyance, water treatment and distribution are all included.

$\mathrm{b}$ : include source extraction and conveyance

c: include source extraction and water treatment

d: El of water supply for billed water, including raw water pumping, conveyance, water treatment and distribution

e: El value for San Diego city.

f: include water treatment and distribution

g: include conveyance and water treatment

$\mathrm{h}$ : include raw water pumping, conveyance, and water distribution 
Table S2b Summary of energy intensity values $\left(\mathrm{kWh} / \mathrm{m}^{3}\right)$ for wastewater system by water sources and end use sectors $(\mathrm{sf}=\mathrm{surface}$ water, gw $=$ groundwater, $\mathrm{ww}=$ wastewater, ag = agricultural, ind = industrial, mun = municipal, $\mathrm{NS}=$ not specified, $\mathrm{L}=\mathrm{low}, \mathrm{H}=\mathrm{high}$ ).

\begin{tabular}{|c|c|c|c|c|c|c|c|}
\hline & \multicolumn{3}{|c|}{ ww collection } & \multicolumn{2}{|c|}{ ww treatment } & \multirow{2}{*}{$\begin{array}{l}\text { ww discharge } \\
\text { NS } \\
\end{array}$} & \multirow[t]{2}{*}{ Study region } \\
\hline & NS & mun & NS & ind & mun & & \\
\hline Burt et al. 2011 & & & & & & & California, USA \\
\hline Burton 1996 & & $0.04-0.05$ & & & $0.18-0.78^{\mathrm{a}}$ & & USA \\
\hline Carlson and Wallburger 2007 & $0.01-0.26$ & & & & & & USA \\
\hline CEC 2005 & 0.04 & & $0.29-1.22^{\mathrm{a}}$ & & & $0-0.11$ & California, USA \\
\hline CEC 2006 & 0.04 & & & & $0.25-0.51$ & & California, USA \\
\hline Cheng 2002 & & & & & 0.41 & & Taiwan \\
\hline Cohen et al. 2004 & & & 0.46 & & $0.11-0.8$ & & California, USA \\
\hline daMonsabert et al. 2008 & & 0.25 & & & & & USA \\
\hline \multirow[t]{7}{*}{ Dhakal et al. 2015} & & & & & $0.1-0.82$ & & Australia \\
\hline & & & & & $0.29-1.22$ & & USA \\
\hline & & & & & $0.39-0.83^{\mathrm{a}}$ & & Germany \\
\hline & & & & & & 0.09 & Singapore \\
\hline & & & & & $0.09-0.2^{\mathrm{a}}$ & & Bangkok, Thailand \\
\hline & & & & & $0.11^{\mathrm{a}}$ & & Delhi, India \\
\hline & & & & & $0.19-1.1^{\mathrm{a}}$ & & Tokyo, Japan \\
\hline EPA 2008 & & & 0.46 & & & & Massachusetts, USA \\
\hline EPRI 2002 & & & $0.177-0.78$ & 0.66 & $0.41^{\mathrm{a}}$ & $0-0.1$ & USA \\
\hline EPRI 2013 & & 0.06 & & & $0.2-0.78^{a}$ & & USA \\
\hline Gans et al. 2007 & & & & & 0.26 & & Beijing, China \\
\hline \multirow[t]{2}{*}{ Jonasson 2007} & & & & & 0.3 & & Austria \\
\hline & & & & & 0.48 & & Sweden \\
\hline \multirow[t]{2}{*}{ Kenway et al. 2008} & & & & & 0.27 & & Gold Coast, Australia \\
\hline & & & & & 0.3 & & Melbourne, Australia \\
\hline
\end{tabular}


Continued Table S2b Summary of energy intensity values $\left(\mathrm{kWh} / \mathrm{m}^{3}\right)$ for wastewater system by water sources and end use sectors $(\mathrm{sf}=$ surface water, gw $=$ groundwater, $\mathrm{ww}=$ wastewater, ag = agricultural, ind = industrial, mun = municipal, NS = not specified, $\mathrm{L}=\mathrm{low}, \mathrm{H}=\mathrm{high}$ ).

\begin{tabular}{|c|c|c|c|c|c|c|c|}
\hline & \multicolumn{2}{|c|}{ ww collection } & \multicolumn{3}{|c|}{ ww treatment } & \multirow{2}{*}{$\begin{array}{l}\text { ww discharge } \\
\text { NS }\end{array}$} & \multirow[t]{2}{*}{ Study region } \\
\hline & NS & mun & NS & ind & mun & & \\
\hline Kenway et al. 2008 & & & & & 0.12 & & Sydney, Australia \\
\hline Kneppers et al. 2009 & & & & & 0.49 & & New Zealand \\
\hline \multirow[t]{2}{*}{ Lazarova et al. 2012} & & & & & $0.24-1.4$ & & Global \\
\hline & & & & & 0.3 & & Strass, Australia \\
\hline \multirow[t]{5}{*}{ Liu et al. 2012} & & & & & & & Egypt \\
\hline & & & & & & & brazil \\
\hline & & & & & & & Russia \\
\hline & & & & & & & India \\
\hline & & & & & & & China \\
\hline M/J Industrial Solutions 2006 & & & & & $0.37-0.6$ & & San Francisco, USA \\
\hline Maas 2009 & & 0.06 & $0.36-0.85$ & & 0.46 & 0.06 & Ontario, Canada \\
\hline Mizuta and Shimada 2009 & & 0.07 & & & 0.32 & 0.06 & Japan \\
\hline Monteith et al. 2007 & & & & & $0.31-0.41$ & & Canada \\
\hline NEWRI 2010 & & & & & $0.44-0.55$ & & Singapore \\
\hline Nouri et al. 2006 & & & 0.3 & & & & Iran \\
\hline Olsen and Larson 2003 & & & & & & & Wisconsin, USA \\
\hline Park and Bennette 2010 & $0-0.23$ & & $0.13-1.2$ & & & $0.26^{\mathrm{c}}$ & California, USA \\
\hline \multirow[t]{6}{*}{ Pheasant and Tait 2010} & & & & & 0.5 & $0.06^{\mathrm{d}}$ & Sweden \\
\hline & & & & & 0.51 & $0.12^{\mathrm{d}}$ & UK \\
\hline & & & & & 0.48 & $0.11^{\mathrm{d}}$ & Netherlands \\
\hline & & & & & 0.59 & $0.14^{\mathrm{d}}$ & Norway \\
\hline & & & & & 0.3 & $0.3^{\mathrm{d}}$ & Australia \\
\hline & & & & & 0.23 & $0.05^{\mathrm{d}}$ & German \\
\hline
\end{tabular}


Continued Table S2b Summary of energy intensity values $\left(\mathrm{kWh} / \mathrm{m}^{3}\right)$ for wastewater system by water sources and end use sectors $(\mathrm{sf}=$ surface water, gw = groundwater, $\mathrm{ww}=$ wastewater, ag = agricultural, ind $=$ industrial, mun = municipal, $\mathrm{NS}=$ not specified, $\mathrm{L}=\mathrm{low}, \mathrm{H}=$ high $)$.

\begin{tabular}{|c|c|c|c|c|c|c|c|}
\hline & & ection & & $\overline{w w t}$ & & ww discharge & Study region \\
\hline & NS & mun & NS & ind & mun & NS & \\
\hline \multirow[t]{4}{*}{ Pheasant and Tait 2010} & & & & & 0.22 & $0.05^{\mathrm{d}}$ & Belgium \\
\hline & & & & & 0.42 & $0.1^{\mathrm{d}}$ & Denmark \\
\hline & & & & & 0.6 & $0.14^{\mathrm{d}}$ & France \\
\hline & & & & & 0.28 & $0.07^{\mathrm{d}}$ & Luxembourg \\
\hline \multirow[t]{2}{*}{ Plappally and Lienhard 2012} & $0-0.04$ & & & & $0.01-1.5$ & $0-0.39$ & USA \\
\hline & & & & & $0.44-1.1$ & & Australia \\
\hline Pirne and Yonkin 2008 & & & & & $0.39^{\mathrm{a}}$ & & New York, USA \\
\hline Radcliffe 2004 & & & & & 0.42 & & New South Wales, Australia \\
\hline SAIC 2006 & & & $0.60-1.93$ & & & & Wisconsin, USA \\
\hline Sauer and Kimber 2003 & & & & & & & Iowa, USA \\
\hline Siddiqi et al. 2011 & & & $0.2-1.2$ & & & $0-0.1$ & Compiled \\
\hline Singh et al. 2012 & & & & & 0.8 & & India \\
\hline Smith 1978 & & & & & $0.61-0.98^{\mathrm{b}}$ & & USA \\
\hline Waughray 2011 & & & 0.66 & & & & USA \\
\hline WEF 2009 & & & & & $0.18-0.78^{\mathrm{a}}$ & & USA \\
\hline WWAP 2014 & & & $0.62-0.87$ & & & & Global \\
\hline L & 0 & 0.04 & 0.04 & & 0.11 & 0 & \\
\hline H & 0.26 & 0.25 & 1.925 & & 0.98 & 0.26 & \\
\hline
\end{tabular}

a: include wastewater collection and treatment

b: include wastewater collection, treatment and disposal

c: value for Santa Rosa, CA

$\mathrm{d}$ : include wastewater collection and discharge 
Table S3 Summary of energy intensity $(\mathrm{EI})$ values $\left(\mathrm{kWh} / \mathrm{m}^{3}\right)$ for desalination by technologies and water sources $(\mathrm{sw}=\mathrm{seawater}$, br $=$ brackish water, $\mathrm{MSF}=$ multistage flash distillation, $\mathrm{MED}=$ multi-effect distillation, $\mathrm{RO}=$ reverse osmosis, $\mathrm{ED}=$ electrodialysis, $\mathrm{NS}=$ not $\mathrm{specified}, \mathrm{L}=$ low, $\mathrm{H}=\mathrm{high}$ ).

\begin{tabular}{|c|c|c|c|c|c|c|c|c|c|c|}
\hline & \multicolumn{5}{|c|}{ membrane } & \multicolumn{4}{|c|}{ Thermal } & \multirow[t]{3}{*}{ Study region } \\
\hline & \multicolumn{2}{|c|}{$\mathrm{RO}$} & \multicolumn{3}{|c|}{ electrodialysis } & \multicolumn{2}{|c|}{ MSF } & \multicolumn{2}{|c|}{ MED } & \\
\hline & $\underline{\mathrm{sw}}$ & $\underline{\mathrm{br}}$ & NS & sw & $\mathrm{br}$ & NS & sw/br & NS & sw/br & \\
\hline Abdel-Jawad 2001 & & & & & & & 16 & & $9.7-13.6$ & GCC \\
\hline Al-Karaghouli and Kazmerski 2011 & & & $0.7-5.5$ & & & $2.5-5^{\mathrm{a}}, 52.8-78.33^{\mathrm{b}}$ & & $2-2.5^{\mathrm{a}}, 40.28-63.89^{\mathrm{b}}$ & & Global \\
\hline Al-Karaghouli et al. 2010 & $5.5-13$ & $0.89-8$ & & & & & & & & Global \\
\hline Blank 2007 & $5 \sim 7$ & & & & & & $2.5-5^{\mathrm{a}}, 40-120^{\mathrm{b}}$ & & $2-2.5^{\mathrm{a}}, 30-120^{\mathrm{b}}$ & Global \\
\hline CEC 2006 & 3.64 & $0.328-1.379$ & & & & & & & & California \\
\hline Charcosset 2009 & $2.51-10.04$ & & & & & & & & & Global \\
\hline Charcosset 2009; Colombo et al. 1999 & $3 \sim 10$ & & & & & & & & & NS \\
\hline Clayton et al. 2014 & & $1 \sim 2.6$ & & & & & & & & Texas, USA \\
\hline Cohen et al. 2004 & $1.11-1.45$ & $0.328-1.379$ & & & & & & & & California \\
\hline El-sayed 2001 & & & & & & & $2-2.2^{\mathrm{a}}, 34-102^{\mathrm{b}}$ & & & NS \\
\hline EPRI 2013 & 3.17 & $0.78-1.585$ & & & & & & & & USA \\
\hline EU 2008 & $4-13$ & $1-3$ & & $1.5-4$ & $0.5-2.5$ & & $2.5-3.5^{\mathrm{a}}, 80.6^{\mathrm{b}}$ & & $1.5-2.5^{\mathrm{a}}, 80.6^{\mathrm{b}}$ & Europe \\
\hline Fritzmann et al. 2007 & $0.4-7$ & $<1$ & & & & & & & & NS \\
\hline Ghaffour et al. 2014 & $2.5-7$ & $0.5-3$ & & & & $10 \sim 16$ & & & & different area \\
\hline Greenlee et al. 2009; Wilf and Klinko 2001 & $3.8-4.8$ & & & & & & & & & NS \\
\hline Greenlee et al. 2009; Wilf and Klinko 2001 & $3.07-3.25$ & & & & & & & & & Mediterranean \\
\hline IAEA 2002 & 5 & & & & & & & & & KANUPP, Pakistan \\
\hline IRENA 2012 & $3.5-5$ & & & & & & & & & Global \\
\hline Lattemann et al 2010 & $2.5-7$ & & & & & & $3-5^{\mathrm{a}}, 69.44-91.67^{\mathrm{b}}$ & & $1.5-2.5^{\mathrm{a}}, 40.27-108.33^{\mathrm{b}}$ & Global \\
\hline \multirow[t]{2}{*}{ Lazarova et al. 2012} & $2.5-5$ & $1-1.5$ & & & & & & & & Global \\
\hline & 2.9 & & & & & & & & & Ashkelon, Israel \\
\hline Mezher et al. 2011 & $4-8$ & $2-3$ & & & & & $3.5-5^{\mathrm{a}}, 44-83^{\mathrm{b}}$ & & $1.5-2.5^{\mathrm{a}}, 27.8-61^{\mathrm{b}}$ & Global \\
\hline National water commission 2008 & $2.8-5$ & $1 \sim 3$ & & & & & $2-3.6^{\mathrm{a}}, 8-10^{\mathrm{b}}$ & & & Global \\
\hline
\end{tabular}


Continued Table S3 Summary of energy intensity $(\mathrm{EI})$ values $\left(\mathrm{kWh} / \mathrm{m}^{3}\right)$ for desalination by technologies and water sources $(\mathrm{sW}=\mathrm{seawater}$, br $=$ brackish water, $\mathrm{MSF}=$ multistage flash distillation, $\mathrm{MED}=$ multi-effect distillation, $\mathrm{RO}=$ reverse osmosis, $\mathrm{ED}=$ electrodialysis, $\mathrm{NS}=$ not $\mathrm{specified}$, $\mathrm{L}=$ low, $\mathrm{H}=$ high).

\begin{tabular}{|c|c|c|c|c|c|c|c|c|c|c|}
\hline & \multicolumn{5}{|c|}{ membrane } & \multicolumn{4}{|c|}{ Thermal } & \multirow[t]{3}{*}{ Study region } \\
\hline & \multicolumn{2}{|c|}{$\mathrm{RO}$} & \multicolumn{3}{|c|}{ electrodialysis } & \multicolumn{2}{|c|}{ MSF } & \multicolumn{2}{|c|}{ MED } & \\
\hline & $\underline{\mathrm{sw}}$ & $\underline{\mathrm{br}}$ & NS & $\mathrm{sw}$ & $\mathrm{br}$ & NS & $\mathrm{sw} / \mathrm{br}$ & NS & $\mathrm{sw} / \mathrm{br}$ & \\
\hline Pfafflin and Ziegler 2006 & & & & 19.00 & $2.4-3.9$ & & & & & NS \\
\hline \multirow[t]{3}{*}{ Plappally and Lienhard 2012} & $3-8.5$ & & & & & & & & & USA \\
\hline & $2.58-5.49$ & $1.02-2.57$ & & & & & & & & California, USA \\
\hline & $3.3-8.5$ & & & & & & & & & Australia \\
\hline Semiat 2008 & & & & & & $1.2-4.5^{\mathrm{a}}, 55-80^{\mathrm{b}}$ & & $1.2-4.5^{\mathrm{a}}, 40-65^{\mathrm{b}}$ & & Global \\
\hline Semiat 2008 & $4 \sim 6$ & & & & & & & & & global \\
\hline Serra et al. 2007 & & & $0.8-1.5$ & & & & & & & Global \\
\hline Sharmila et al. 2004 & 1.85 & & & & & & & & & Kerala, India \\
\hline Tarnacki et al. (2012) and Subramani et al. (2011) & $2-3$ & $1 \sim 2$ & & & & & & & & NS \\
\hline Turek 2003 & & & 6.6 & & & & & & & NS \\
\hline Veza et al. 2001 & & & & $1.48-2.32$ & & & & & & Gran Canaria Island, Spain \\
\hline Vince et al. 2008 & 4.9 & & & & & & & & & NS \\
\hline Wade 2001 & 4.2 & & & & & $3.6^{\mathrm{a}}, 80.56^{\mathrm{b}}$ & & $2.3^{\mathrm{a}}, 71.67^{\mathrm{b}}$ & & NS \\
\hline Water in the west, 2013 & & & & & & 4.75-68.69 & & $39.62-105.68$ & & Global \\
\hline Williams and Simmons 2013 & $2.2-8.5$ & $1-2.5$ & & & & $2.5-5^{\mathrm{a}}, 19.44-77.78^{\mathrm{b}}$ & & $1-3^{\mathrm{a}}, 8.89-20^{\mathrm{b}}$ & & Global \\
\hline Younos and Tulou 2005 & & & 1.70 & & & 42 & & & & Global \\
\hline Younos and Tulou 2005 & $4.2-10$ & & & & & & & & & Global \\
\hline Zander et al 2008 & $2.5-7$ & $0.5-3$ & & & & & & & & USA \\
\hline $\mathbf{L}$ & 0.4 & 0.37 & 0.7 & 1.5 & 0.5 & 10 & 10 & 10 & 9.7 & \\
\hline H & 13 & 8 & 6.6 & 19 & 3.9 & 84 & 125 & 105.7 & 122.5 & \\
\hline
\end{tabular}

a: El for electricity requirement

b: El for thermal energy requirement 
Table S4. Time-involving energy intensity for RO desalination $\left(\mathrm{kWh} / \mathrm{m}^{3}\right)$.

\begin{tabular}{lllllllll}
\hline & $\mathbf{1 9 7 3 -}$ & $\mathbf{1 9 7 8 -}$ & $\mathbf{1 9 8 3 -}$ & $\mathbf{1 9 8 8 -}$ & $\mathbf{1 9 9 3 -}$ & $\mathbf{1 9 9 8 -}$ & $\mathbf{2 0 0 3 -}$ & $\mathbf{2 0 0 8 -}$ \\
& $\mathbf{1 9 7 7}$ & $\mathbf{1 9 8 2}$ & $\mathbf{1 9 8 7}$ & $\mathbf{1 9 9 2}$ & $\mathbf{1 9 9 7}$ & $\mathbf{2 0 0 2}$ & $\mathbf{2 0 0 7}$ & $\mathbf{2 0 1 2}$ \\
\hline seawater & 12 & 7.8 & 6.3 & 4.8 & 4.2 & 3.5 & 2 & 1.8 \\
& & & & & & & & \\
brackish & 6 & 3.9 & 3.15 & 2.4 & 2.1 & 1.75 & 1.5 & 1.25 \\
\hline
\end{tabular}


Table S5. Region-average and time-involving conversion ratio from primary energy to electricity

\begin{tabular}{|c|c|c|c|c|c|c|c|c|}
\hline \multirow{2}{*}{ Region } & 1973- & 1978- & 1983- & 1988- & 1993- & 1998- & 2003 & 2008 \\
\hline & 1977 & 1982 & 1987 & 1992 & 1997 & 2002 & 2007 & 2012 \\
\hline USA & 3.05 & 3.05 & 3.00 & 2.98 & 2.91 & 2.92 & 2.87 & 2.81 \\
\hline China & 3.39 & 3.61 & 3.38 & 3.41 & 3.44 & 3.34 & 3.27 & 2.98 \\
\hline India & 3.30 & 3.28 & 3.81 & 3.90 & 4.14 & 4.65 & 4.60 & 4.42 \\
\hline Middle East & 3.88 & 4.30 & 4.05 & 3.91 & 4.24 & 3.78 & 3.76 & 3.70 \\
\hline Western Europe & 2.86 & 2.85 & 2.91 & 2.84 & 2.75 & 2.67 & 2.61 & 2.51 \\
\hline Former Soviet Union & 4.28 & 4.23 & 4.40 & 4.14 & 3.84 & 3.82 & 3.73 & 3.50 \\
\hline Latin America & 2.94 & 2.87 & 2.93 & 3.34 & 3.25 & 3.20 & 3.28 & 3.15 \\
\hline Southeast Asia & 2.88 & 3.06 & 3.18 & 3.11 & 3.05 & 3.05 & 2.95 & 2.94 \\
\hline Canada & 2.14 & 2.46 & 2.77 & 3.00 & 3.00 & 2.81 & 2.97 & 2.80 \\
\hline Australia \& NZ & 3.26 & 3.12 & 2.95 & 2.87 & 2.80 & 3.04 & 3.04 & 3.03 \\
\hline Eastern Europe & 3.72 & 3.59 & 3.68 & 3.82 & 3.85 & 3.57 & 3.39 & 3.29 \\
\hline Korea & 3.28 & 3.10 & 3.22 & 3.12 & 2.88 & 2.83 & 2.75 & 2.79 \\
\hline Japan & 2.77 & 2.81 & 2.82 & 2.72 & 2.75 & 2.70 & 2.65 & 2.61 \\
\hline Africa & 3.38 & 3.00 & 3.13 & 3.04 & 3.18 & 3.05 & 2.99 & 3.10 \\
\hline
\end{tabular}


Table S6. Time-involving and region-specific percent of industrial water withdrawal for power plant cooling.

\begin{tabular}{lllllllll}
\hline Region & $\mathbf{1 9 7 3 -}$ & $\mathbf{1 9 7 8 -}$ & $\mathbf{1 9 8 3 -}$ & $\mathbf{1 9 8 8 -}$ & $\mathbf{1 9 9 3 -}$ & $\mathbf{1 9 9 8 -}$ & $\mathbf{2 0 0 3 -}$ & $\mathbf{2 0 0 8 -}$ \\
& $\mathbf{1 9 7 7}$ & $\mathbf{1 9 8 2}$ & $\mathbf{1 9 8 7}$ & $\mathbf{1 9 9 2}$ & $\mathbf{1 9 9 7}$ & $\mathbf{2 0 0 2}$ & $\mathbf{2 0 0 7}$ & $\mathbf{2 0 1 2}$ \\
\hline USA & $83.61 \%$ & $83.61 \%$ & $83.61 \%$ & $83.61 \%$ & $84.51 \%$ & $85.42 \%$ & $86.32 \%$ & $86.32 \%$ \\
China & $30.86 \%$ & $30.86 \%$ & $30.86 \%$ & $30.86 \%$ & $36.35 \%$ & $41.85 \%$ & $47.34 \%$ & $47.34 \%$ \\
India & $57.04 \%$ & $57.04 \%$ & $57.04 \%$ & $57.04 \%$ & $59.55 \%$ & $62.06 \%$ & $64.58 \%$ & $64.58 \%$ \\
Middle East & $22.88 \%$ & $22.88 \%$ & $22.88 \%$ & $22.88 \%$ & $26.14 \%$ & $29.39 \%$ & $32.65 \%$ & $32.65 \%$ \\
Western Europe & $59.01 \%$ & $59.01 \%$ & $59.01 \%$ & $59.01 \%$ & $60.33 \%$ & $61.65 \%$ & $62.97 \%$ & $62.97 \%$ \\
Former Soviet Union & $51.66 \%$ & $51.66 \%$ & $51.66 \%$ & $51.66 \%$ & $53.93 \%$ & $56.21 \%$ & $58.48 \%$ & $58.48 \%$ \\
Latin America & $10.98 \%$ & $10.98 \%$ & $10.98 \%$ & $10.98 \%$ & $12.95 \%$ & $14.92 \%$ & $16.88 \%$ & $16.88 \%$ \\
Southeast Asia & $10.52 \%$ & $10.52 \%$ & $10.52 \%$ & $10.52 \%$ & $13.35 \%$ & $16.19 \%$ & $19.03 \%$ & $19.03 \%$ \\
Canada & $80.59 \%$ & $80.59 \%$ & $80.59 \%$ & $80.59 \%$ & $80.77 \%$ & $80.94 \%$ & $81.12 \%$ & $81.12 \%$ \\
Australia \& NZ & $40.29 \%$ & $40.29 \%$ & $40.29 \%$ & $40.29 \%$ & $41.30 \%$ & $42.32 \%$ & $43.33 \%$ & $43.33 \%$ \\
Eastern Europe & $46.95 \%$ & $46.95 \%$ & $46.95 \%$ & $46.95 \%$ & $51.52 \%$ & $56.09 \%$ & $60.66 \%$ & $60.66 \%$ \\
Korea & $1.61 \%$ & $1.61 \%$ & $1.61 \%$ & $1.61 \%$ & $3.13 \%$ & $4.65 \%$ & $6.16 \%$ & $6.16 \%$ \\
Japan & $4.60 \%$ & $4.60 \%$ & $4.60 \%$ & $4.60 \%$ & $6.25 \%$ & $7.89 \%$ & $9.54 \%$ & $9.54 \%$ \\
Africa & $40.38 \%$ & $40.38 \%$ & $40.38 \%$ & $40.38 \%$ & $42.25 \%$ & $44.12 \%$ & $45.99 \%$ & $45.99 \%$ \\
\hline & & & & & & & &
\end{tabular}


Table S7. Estimate of time-involving and region-specific seawater withdrawal for power plant cooling (bcm/yr).

\begin{tabular}{lllllllll}
\hline Region & $\mathbf{1 9 7 3 -}$ & $\mathbf{1 9 7 8 -}$ & $\mathbf{1 9 8 3 -}$ & $\mathbf{1 9 8 8}-$ & $\mathbf{1 9 9 3 -}$ & $\mathbf{1 9 9 8 -}$ & $\mathbf{2 0 0 3 -}$ & $\mathbf{2 0 0 8 -}$ \\
& $\mathbf{1 9 7 7}$ & $\mathbf{1 9 8 2}$ & $\mathbf{1 9 8 7}$ & $\mathbf{1 9 9 2}$ & $\mathbf{1 9 9 7}$ & $\mathbf{2 0 0 2}$ & $\mathbf{2 0 0 7}$ & $\mathbf{2 0 1 2}$ \\
\hline USA & 35.70 & 40.32 & 32.12 & 31.48 & 32.68 & 33.88 & 35.87 & 29.57 \\
China & 4.76 & 4.91 & 4.11 & 5.40 & 9.01 & 11.36 & 10.54 & 10.74 \\
India & 3.75 & 3.11 & 2.72 & 3.70 & 3.22 & 2.68 & 3.76 & 4.74 \\
Middle East & 11.47 & 12.54 & 13.60 & 31.56 & 66.21 & 101.67 & 123.99 & 140.00 \\
Western Europe & 15.94 & 16.51 & 16.61 & 16.24 & 15.63 & 15.29 & 13.83 & 12.02 \\
Former Soviet Union & 5.36 & 5.56 & 5.46 & 5.09 & 4.80 & 4.53 & 4.54 & 4.38 \\
Latin America & 1.45 & 1.60 & 1.76 & 2.03 & 2.41 & 2.89 & 3.55 & 4.21 \\
Southeast Asia & 4.62 & 5.35 & 6.19 & 7.26 & 13.14 & 22.69 & 49.98 & 55.54 \\
Canada & 2.69 & 2.82 & 2.94 & 2.98 & 2.99 & 3.00 & 3.02 & 3.05 \\
Australia \& NZ & 1.45 & 1.52 & 1.66 & 1.78 & 1.91 & 1.90 & 2.02 & 2.31 \\
Eastern Europe & 2.08 & 2.32 & 2.40 & 2.08 & 1.95 & 1.78 & 1.87 & 1.73 \\
Korea & 6.68 & 7.22 & 7.61 & 8.00 & 8.93 & 10.25 & 11.65 & 12.51 \\
Japan & 83.34 & 86.70 & 84.86 & 83.03 & 87.96 & 98.45 & 107.30 & 108.44 \\
Africa & 1.15 & 1.25 & 1.47 & 1.61 & 1.80 & 1.91 & 2.22 & 2.48 \\
\hline & & & & & & & & \\
\hline
\end{tabular}


Table S8. Region-specific energy for water (EJ/yr) at water sources, water-use processes and end-use sectors levels in 2010 ( $\operatorname{surf}=$ surface water, $\mathrm{gw}=$ groundwater, non-fresh $=$ non-fresh water, $\mathrm{sc}=$ source and conveyance, $\operatorname{trt}=$ water treatment, dist $=$ distribution, ww_clt $=$ wastewater collection, $w$ w_trt $=$ wastewater treatment, $w w \_d i s=$ wastewater discharge, $\mathrm{ag}=$ agriculture, ind $=$ industry, mun $=$ municipal).

\begin{tabular}{|c|c|c|c|c|c|c|c|c|c|c|c|c|}
\hline & \multicolumn{3}{|c|}{ water sources } & \multicolumn{6}{|c|}{ water-use processes } & \multicolumn{3}{|c|}{ end-use sectors } \\
\hline & surf & gw & non-fresh & sc & trt & dist & ww_clt & ww_trt & ww_dis & $\underline{\mathrm{ag}}$ & ind & mun \\
\hline USA & 0.928 & 0.125 & 0.059 & 0.319 & 0.237 & 0.158 & 0.039 & 0.342 & 0.039 & 0.141 & 0.302 & 0.691 \\
\hline China & 0.936 & 0.395 & 0.001 & 0.548 & 0.324 & 0.171 & 0.027 & 0.246 & 0.026 & 0.328 & 0.489 & 0.526 \\
\hline India & 0.999 & 0.639 & 0.000 & 1.180 & 0.212 & 0.200 & 0.005 & 0.041 & 0.005 & 1.039 & 0.062 & 0.542 \\
\hline $\begin{array}{l}\text { Middle East } \\
\text { Western }\end{array}$ & 0.130 & 0.496 & 0.602 & 0.520 & 0.671 & 0.060 & 0.007 & 0.066 & 0.007 & 0.349 & 0.407 & 0.573 \\
\hline $\begin{array}{l}\text { Europe } \\
\text { Former Soviet }\end{array}$ & 0.710 & 0.288 & 0.032 & 0.216 & 0.247 & 0.121 & 0.041 & 0.379 & 0.039 & 0.045 & 0.533 & 0.466 \\
\hline Union & 0.390 & 0.234 & 0.094 & 0.140 & 0.247 & 0.074 & 0.023 & 0.215 & 0.022 & 0.044 & 0.353 & 0.324 \\
\hline Latin America & 0.482 & 0.308 & 0.023 & 0.317 & 0.237 & 0.146 & 0.011 & 0.095 & 0.010 & 0.152 & 0.189 & 0.475 \\
\hline Southeast Asia & 0.443 & 0.291 & 0.003 & 0.373 & 0.180 & 0.114 & 0.010 & 0.092 & 0.010 & 0.242 & 0.225 & 0.313 \\
\hline Canada & 0.126 & 0.014 & 0.000 & 0.031 & 0.023 & 0.014 & 0.007 & 0.060 & 0.006 & 0.003 & 0.078 & 0.060 \\
\hline $\begin{array}{l}\text { Australia \& NZ } \\
\text { Eastern }\end{array}$ & 0.050 & 0.020 & 0.003 & 0.021 & 0.020 & 0.012 & 0.002 & 0.018 & 0.002 & 0.010 & 0.023 & 0.043 \\
\hline Europe & 0.139 & 0.055 & 0.001 & 0.041 & 0.049 & 0.022 & 0.008 & 0.069 & 0.007 & 0.003 & 0.113 & 0.079 \\
\hline Korea & 0.094 & 0.022 & 0.000 & 0.033 & 0.026 & 0.017 & 0.005 & 0.044 & 0.005 & 0.005 & 0.059 & 0.065 \\
\hline Japan & 0.185 & 0.062 & 0.004 & 0.079 & 0.057 & 0.036 & 0.011 & 0.101 & 0.011 & 0.009 & 0.136 & 0.151 \\
\hline Africa & 0.281 & 0.129 & 0.140 & 0.192 & 0.221 & 0.074 & 0.006 & 0.053 & 0.006 & 0.149 & 0.099 & 0.304 \\
\hline Globe & 5.892 & 3.077 & 0.963 & 4.011 & 2.750 & 1.219 & 0.203 & 1.821 & 0.195 & 2.518 & 3.070 & 4.611 \\
\hline
\end{tabular}


Table S9. Region-specific water withdrawal (bcm/yr) at water sources, water-use processes and end-use sectors levels in 2010 ( $\operatorname{surf}=$ surface water, $\mathrm{gw}=$ groundwater, non-fresh $=$ non-fresh water, $\mathrm{sc}=$ source and conveyance, $\operatorname{trt}=$ water treatment, dist $=$ distribution, ww_clt $=$ wastewater collection, $w$ w_trt $=$ wastewater treatment, $w w \_d i s=$ wastewater discharge, $\mathrm{ag}=$ agriculture, ind $=$ industry, mun $=$ municipal).

\begin{tabular}{|c|c|c|c|c|c|c|c|c|c|c|c|c|}
\hline & \multicolumn{3}{|c|}{ water sources } & \multicolumn{6}{|c|}{ water-use processes } & \multicolumn{3}{|c|}{ end-use sectors } \\
\hline & surf & gw & non-fresh & sc & trt & dist & ww_clt & ww_trt & ww_dis & $\underline{\mathrm{ag}}$ & ind & mun \\
\hline USA & 316.2 & 104.4 & 30.2 & 211.4 & 89.5 & 63.4 & 47.8 & 45.4 & 45.4 & 175.3 & 181.8 & 64.0 \\
\hline China & 556.8 & 108.8 & 10.8 & 404.5 & 163.8 & 64.6 & 32.3 & 18.8 & 18.8 & 460.5 & 135.5 & 71.1 \\
\hline India & 396.5 & 251.0 & 4.7 & 751.2 & 66.2 & 50.8 & 7.6 & 4.4 & 4.4 & 688.0 & 17.0 & 56.0 \\
\hline $\begin{array}{l}\text { Middle East } \\
\text { Western }\end{array}$ & 61.7 & 173.2 & 143.2 & 311.9 & 40.8 & 18.2 & 5.4 & 3.6 & 3.6 & 209.9 & 16.2 & 19.8 \\
\hline $\begin{array}{l}\text { Europe } \\
\text { Former Soviet }\end{array}$ & 192.4 & 64.4 & 12.3 & 143.3 & 123.7 & 54.2 & 35.6 & 31.2 & 31.2 & 91.7 & 128.1 & 54.3 \\
\hline Union & 182.6 & 31.0 & 5.2 & 104.5 & 68.2 & 23.7 & 23.3 & 11.4 & 11.4 & 135.1 & 62.3 & 25.3 \\
\hline $\begin{array}{l}\text { Latin America } \\
\text { Southeast }\end{array}$ & 251.4 & 81.2 & 4.4 & 187.6 & 98.5 & 52.0 & 21.6 & 9.2 & 9.2 & 241.9 & 39.8 & 55.5 \\
\hline Asia & 455.7 & 135.5 & 55.6 & 228.0 & 92.2 & 43.6 & 26.7 & 7.6 & 7.6 & 518.0 & 50.3 & 49.4 \\
\hline Canada & 37.1 & 1.7 & 3.1 & 14.6 & 12.0 & 5.5 & 5.8 & 4.9 & 4.9 & 4.7 & 28.5 & 5.5 \\
\hline $\begin{array}{l}\text { Australia \& NZ } \\
\text { Eastern }\end{array}$ & 15.5 & 5.3 & 2.3 & 17.3 & 7.6 & 4.6 & 2.2 & 2.0 & 2.0 & 12.8 & 3.9 & 4.6 \\
\hline Europe & 33.9 & 6.1 & 1.7 & 20.3 & 21.7 & 7.7 & 5.7 & 3.9 & 3.9 & 4.3 & 27.6 & 8.2 \\
\hline Korea & 26.3 & 2.9 & 12.5 & 31.7 & 13.6 & 6.8 & 7.2 & 4.2 & 4.2 & 16.1 & 6.2 & 7.0 \\
\hline Japan & 71.8 & 9.4 & 108.5 & 87.1 & 28.3 & 15.4 & 12.0 & 11.6 & 11.6 & 54.4 & 11.6 & 15.4 \\
\hline Africa & 159.5 & 45.2 & 3.3 & 148.6 & 45.2 & 26.8 & 40.3 & 6.4 & 6.4 & 224.6 & 12.7 & 34.1 \\
\hline Globe & 2757.4 & 1020.2 & 397.8 & 2662.0 & 871.3 & 437.3 & 273.4 & 164.7 & 164.7 & 2837.4 & 721.5 & 470.2 \\
\hline
\end{tabular}




\section{Appendix S1}

Downloadable data for country-level key variables and estimated E4W for 2010 (see attached Excel file) 


\section{References}

1. Shepard, D. In A two-dimensional interpolation function for irregularly-spaced data, Proceedings of the 1968 23rd ACM national conference, 1968; ACM: 1968; pp 517-524.

2. Food and Agriculture Organization (FAO), AQUASTAT database. $<$ http://www.fao.org/nr/water/aquastat/dbases/index.stm>, date of access: 02/15/2016.

3. Murray, R. C.; Reeves, B. E., Estimated use of water in the United States in 1975. U.S. Geological Survey: Arlington, Virginia, 1977.

4. Solley, W. B.; Chase, E. B.; Mann IV, W. B. Estimated use of water in the United States in 1980; 2330-5703; Geological Survey (US): 1983.

5. Solley, W. B.; Merk, C. F.; Pierce, R. R., Estimated Use of Water in the United States in 1985. U.S. Geological Survey: Denver, Colorado, 1988.

6. Solley, W. B.; Pierce, R. R.; Perlman, H. A., Estimated use of water in the United States in 1990. U.S. Geological Survey: 1993.

7. Solley, W. B.; Pierce, R. R.; Perlman, H. A., Estimated use of water in the United States in 1995. US Geological Survey: 1998.

8. Hutson, S. S.; Barber, N. L.; Kenny, J. F.; Linsey, K. S.; Lumia, D. S.; Maupin, M. A., Estimated use of water in the United States in 2000. Geological Survey (USGS): 2004.

9. Kenny, J. F.; Barber, N. L.; Hutson, S. S.; Linsey, K. S.; Lovelace, J. K.; Maupin, M. A., Estimated use of water in the United States in 2005. US Geological Survey: 2009.

10. Maupin, M. A.; Kenny, J. F.; Hutson, S. S.; Lovelace, J. K.; Barber, N. L.; Linsey, K. S. Estimated use of water in the United States in 2010; 2330-5703; US Geological Survey: 2014.

11. Siebert, S. Kummu, M., Porkka, M., Döll, P., Ramankutty, N., \& Scanlon, B. R.A global data set of the extent of irrigated land from 1900 to 2005. Hydrology and Earth System Sciences 19, 1521-1545 (2015).

12. The World Bank, GDP at market prices (current US\$). $<$ http://data.worldbank.org/indicator/NY.GDP.MKTP.CD>, date of access: 10/15/2016.

13. The World Bank, Industry, value added (\% of GDP). $<$ http://data.worldbank.org/indicator/SH.H2O.SAFE.ZS>, date of access: 10/15/2016.

14. The World Bank, Improved water source (\% of population with access). $<\mathrm{http}$ //data.worldbank.org/indicator/NV.IND.TOTL.ZS >, date of access: 10/15/2016.

15. The World Bank, Population, total. <http://data.worldbank.org/indicator/SP.POP.TOTL $>$, date of access: 10/15/2016.

16. Kim, S., M. H., Liu, L., Calvin, K., Clarke, L., Edmonds, J., Kyle, P., Patel, P., Wise, M., Davies, E., Balancing global water availability and use at basin scale in an integrated assessment model. Climatic Change, 2016, 1-15.

17. Central Ground Water Board Board, Ground Water Year Book - India 2010-11. Ministry of Water Resources: Faridabad, India, 2011.

18. Gandhi, V. P.; Bhamoriya, V. Groundwater Irrigation in India; 2011; p 90.

19. Electric Power Research Institute (EPRI), Water \& sustainability (volume 4): US electricity consumption for water supply \& treatment-the next half century. Electric Power Research Institute: 2002.

20. Davies, E. G.; Kyle, P.; Edmonds, J. A., An integrated assessment of global and regional water demands for electricity generation to 2095. Advances in Water Resources 2013, 52, 296-313. 
21. Ghaffour, N.; Lattemann, S.; Missimer, T.; Ng, K. C.; Sinha, S.; Amy, G., Renewable energy-driven innovative energy-efficient desalination technologies. Applied Energy 2014, 136, 1155-1165.

22. Clayton, M. E.; Stillwell, A. S.; Webber, M. E., Implementation of Brackish Groundwater Desalination Using Wind-Generated Electricity: A Case Study of the Energy-Water Nexus in Texas. Sustainability 2014, 6, (2), 758-778.

23. United Nations World Water Assessment Programme (WWAP), The United Nations World Water Development Report 2014: Water and Energy. In UNESCO, Ed. Paris, 2014; Vol. 1.

24. Williams, E. D.; Simmons, J. E., Water in the energy industry. An introduction. BP International Ltd.: 2013.

25. Water in the West, Water and Energy Nexus: A Literature Review; Standford University: Stanford, 2013.

26. Electric Power Research Institute (EPRI), Electricity Use and Management in the Municipal Water Supply and Wastewater Industries; Electric Power Research Institute: Palo Alto, CA, 2013.

27. Tarnacki, K.; Meneses, M.; Melin, T.; van Medevoort, J.; Jansen, A., Environmental assessment of desalination processes: Reverse osmosis and Memstill®. Desalination 2012, 296, 69-80.

28. Plappally, A. L. J., Energy requirements for water production, treatment, end use, reclamation, and disposal. Renewable and Sustainable Energy Reviews 2012, 16, (7), 4818-4848.

29. Liu, F.; Ouedraogo, A.; Manghee, S.; Danilenko, A., A Primer on Energy Efficiency for Municipal Water and Wastewater Utilities. 2012.

30. International Renewable Energy Agency (IRENA), I. R. E. A. Water Desalination Using Renewable Energy; IRENA: 2012.

31. Waughray, D., Water security: the water-food-energy-climate nexus. Island Press: 2011.

32. Subramani, A.; Badruzzaman, M.; Oppenheimer, J.; Jacangelo, J. G., Energy minimization strategies and renewable energy utilization for desalination: a review. Water research 2011, 45, (5), 1907-1920.

33. Siddiqi, A.; Anadon, L. D., The water-energy nexus in Middle East and North Africa. Energy Policy 2011, 39, (8), 4529-4540.

34. Burt, C. P. E.; Howes, D. J.; Freeman, B. AGRICULTURAL WATER ENERGY EFFICIENCY; Irrigation Training and Research Center: San Luis Obispo, CA, 2011.

35. Al-Karaghouli, A. A.; Kazmerski, L., Renewable energy Opportunities in water desalination. INTECH Open Access Publisher: 2011.

36. Park, L.; Bennett, B. Embedded Energy in Water Studies-Study 2: Water Agency and Function Component Study and Embedded Energy-Water Load Profiles; 2010.

37. Lattemann, S.; Kennedy, M. D.; Schippers, J. C.; Amy, G., Global desalination situation. Sustainability Science and Engineering 2010, 2, 7-39.

38. Al-Karaghouli, A.; Renne, D.; Kazmerski, L. L., Technical and economic assessment of photovoltaicdriven desalination systems. Renewable Energy 2010, 35, (2), 323-328.

39. Maas, C., Greenhouse gas and energy co-benefits of water conservation. POLIS Project on Ecological Governance: 2009.

40. Kneppers, B.; Birchfield, D.; Lawton, M., Energy-water relationships in reticulated water infrastructure systems. Water Supply (kWh/Capita) 2009, 76, (20.86), 75.15.

41. Greenlee, L. F.; Lawler, D. F.; Freeman, B. D.; Marrot, B.; Moulin, P., Reverse osmosis desalination: water sources, technology, and today's challenges. Water research 2009, 43, (9), 2317-2348. 
42. Charcosset, C., A review of membrane processes and renewable energies for desalination. Desalination 2009, 245, (1), 214-231.

43. Water Environment Federation (WEF), Energy Conservation in Water and Wastewater Treatment Facilities. WEF Press: Alexandria, Virginia, 2009.

44. Zander, A.; Elimelech, M.; Furukawa, D.; Gleick, P.; Herd, K.; Jones, K. L.; Rolchigo, P.; Sethi, S.; Tonner, J.; Vaux, H. J., Desalination: A national perspective. National Research Council, The National Academies 2008.

45. Vince, F.; Aoustin, E.; Bréant, P.; Marechal, F., LCA tool for the environmental evaluation of potable water production. Desalination 2008, 220, (1), 37-56.

46. Semiat, R., Energy issues in desalination processes. Environmental science \& technology 2008, 42, (22), 8193-8201.

47. Pirne, M.; Yonkin, M., Statewide assessment of energy use by the municipal water and wastewater sector. New York State Energy Research and Development Authority, Albany 2008.

48. deMonsabert, S. M.; Bakhshi, A.; Headley, J. L., Embodied Energy in Municipal Water and Wastewater. Proceedings of the Water Environment Federation 2008, 2008, (6), 202-222.

49. Commission, N. W. Emerging trends in desalination: A review; Canberra, Australia, 2008.

50. European Union (EU), ADIRA Handbook - A guide to autonomous desalination system concepts; ISBN 978-975-561-311-6; 2008.

51. Environmental Protection Agency (EPA), E. P. A. Ensuring a Sustainable Future: An Energy Management Guidebook for Water and Wastewater Utilities; EPA 832-R-08-002; 2008.

52. Serra, L.; Uche, J.; Raluy, G. In Desalination as a sustainable source of fresh water provision, Proceedings of the 3rd Dubrovnik Conference on Sustainable Development of Energy, Water and Environment Systems, Edited by N. Afgan, Z. Bogdan, N. Duic \& Z. Guzovic, 2007; 2007.

53. Fritzmann, C.; Löwenberg, J.; Wintgens, T.; Melin, T., State-of-the-art of reverse osmosis desalination. Desalination 2007, 216, (1), 1-76.

54. Carlson, S. W.; Walburger, A., Energy index development for benchmarking water and wastewater utilities. Awwa Research Foundation: 2007.

55. Blank, J.; Tusel, G.; Nisanc, S., The real cost of desalted water and how to reduce it further. Desalination 2007, 205, (1), 298-311.

56. Pfafflin, J. R.; Ziegler, E. N., Encyclopedia of environmental science and engineering. fifth ed.; CRC press: 2006; Vol. 1.

57. Science Applications International Corporation (SAIC), Water and Wastewater Energy Best Practice Guide; Division of Energy of State of Wisconsin: Wisconsin, 2006.

58. California Energy Commission (CEC), REFINING ESTIMATES OF WATER-RELATED ENERGY USE IN CALIFORNIA; California Energy Commission: 2006.

59. Younos, T.; Tulou, K. E., Energy needs, consumption and sources. Journal of Contemporary Water Research \& Education 2005, 132, (1), 27-38.

60. California Energy Commission (CEC), California's water-energy relationship.

61. Cohen, R.; Wolff, G.; Nelson, B., Energy Down the Drain. Water Supply 2004.

62. Turek, M., Cost effective electrodialytic seawater desalination. Desalination 2003, 153, (1), 371-376.

63. Sauer, P.; Kimber, A., Energy consumption and costs to treat water and waste water in Iowa. Iowa Association of Municipal Utilities, Ankeny, IA 2003. 
64. Olsen, S. L., Alan In Opportunities and Barriers in Madison, Wisconsin: Understanding Process Energy Use in a Large Municipal Water Utility, Proceedings of ACEEE Summer Study on Energy Efficiency in Industry - Sustainability and Industry: Increasing Energy Efficiency and Reducing Emissions. , New York, 2003; New York, 2003.

65. Elliott, T.; Zeier, B.; Xagoraraki, I.; Harrington, G. W., Energy use at Wisconsin's drinking water facilities. Department of Civil and Environmental Engineering, University of Wisconsin-Madison, Report 2003, 222-1.

66. Wilf, M.; Klinko, K., Optimization of seawater RO systems design. Desalination 2001, 138, (1), 299306.

67. Wade, N. M., Distillation plant development and cost update. Desalination 2001, 136, (1), 3-12.

68. Veza, J. M.; Peñate, B.; Castellano, F., Electrodialysis desalination designed for wind energy (on-grid tests). Desalination 2001, 141, (1), 53-61.

69. El-Sayed, Y., Designing desalination systems for higher productivity. Desalination 2001, 134, (1), 129-158.

70. Abdel-Jawad, M., Energy Sources for coupling with desalination plants in the GCC countries. consultancy report prepared for ESCWA 2001.

71. Colombo, D.; De Gerloni, M.; Reali, M., An energy-efficient submarine desalination plant. Desalination 1999, 122, (2), 171-176.

72. Burton, F. L., Water and wastewater industries: characteristics and energy management opportunities. In Palo Alto, CA: Electric Power Research Institute: 1996.

73. Smith, R. Total energy consumption for municipal wastewater treatment; EPA-600/2-78-149; US Environmental Protection Agency, Office of Research and Development, Municipal Environmental Research Laboratory: Cincinnati OH, 1978.

74. Gans, N., Mobini, S. \& Zhang, X. Mass and Energy Balances at the Gaobeidian Wastewater Treatment Plant in Beijing, China Master thesis, Lund University, (2007).

75. Raluy, R. G., Serra, L., Uche, J. \& Valero, A. Life Cycle Assessment of Water Production Technologies-Part 2: Reverse Osmosis Desalination versus the Ebro River Water Transfer ( 9 pp). The International Journal of Life Cycle Assessment 10, 346-354 (2005).

76. Singh, P., Carliell-Marquet, C. \& Kansal, A. Energy pattern analysis of a wastewater treatment plant. Applied Water Science 2, 221-226 (2012).

77. Dhakal, S., Shrestha, S., Shrestha, A., Kaneko, S. \& Kansal, A. Water-Energy-Carbon Nexus in Cities: Cases from Bangkok, New Delhi, Tokyo. (Asian Institute of Technology, Paris, 2015).

78. Shah, T., Scott, C., Kishore, A. \& Sharma, A. Energy-irrigation nexus in South Asia: improving groundwater conservation and power sector viability. Vol. 70 (IWMI, 2004).

79. Cheng, C.-L. Study of the inter-relationship between water use and energy conservation for a building. Energy and buildings 34, 261-266 (2002).

80. Costa, A. R. \& De Pinho, M. N. Performance and cost estimation of nanofiltration for surface water treatment in drinking water production. Desalination 196, 55-65 (2006).

81. Lazarova, V., Choo, K.-H. \& Cornel, P. Water-energy interactions in water reuse. (IWA Publishing, 2012).

82. Jonasson, M. Energy Benchmark for Wastewater Treatment Processes - a comparison between Sweden and Austria Master thesis, University of Innsbruck, (2007). 
83. Kenway, S. J., Priestley, A., Cook, S., Seo, S., Inman, M., Gregory, A., Hall, M. Energy use in the provision and consumption of urban water in Australia and New Zealand. Water Services Association of Australia (WSAA): Sydney, Australia (2008).

84. Solutions, M. J. I. Municipal wastewater treatment plant energy baseline study. (Pacific Gas and Electric Company, San Francisco, 2006).

85. Mizuta, K. \& Shimada, M. Benchmarking energy consumption in municipal wastewater treatment plants in Japan. Water Science \& Technology 62 (2010).

86. Monteith, H., Kalogo, Y. \& Louzeiro, N. ACHIEVING STRINGENT EFFLUENT LIMITS TAKES A LOT OF ENERGY! Proceedings of the Water Environment Federation 2007, 4343-4356 (2007).

87. NEWRI. Water \& Energy in The Urban Water Cycle - improving energy efficiency in Municipal Wastewater Treatment. (2010).

88. Nouri, J. Jafarinia, M., Naddafi, K., Nabizadeh, R., Mahvi, A. H., \& Nouri, N. Energy recovery from wastewater treatment plant. Pakistan Journal of Biological Sciences 9, 3-6 (2006).

89. Plappally, A. K. \& Lienhard, J. H. Costs for water supply, treatment, end-use and reclamation. Desalination and Water Treatment 51, 200-232 (2012).

90. Radcliffe, J. C. Water recycling in Australia: a review undertaken by the Australian academy of technological sciences and engineering. (Australian Academy of Technological Sciences and Engineering, 2004).

91. IAEA. Status of design concepts of nuclear desalination plants. (International Atomic Energy Agency, Vienna, Austria 2002).

92. Mezher, T., Fath, H., Abbas, Z. \& Khaled, A. Techno-economic assessment and environmental impacts of desalination technologies. Desalination 266, 263-273 (2011).

93. Sharmila, N., Jalihal, P., Swamy, A. \& Ravindran, M. Wave powered desalination system. Energy 29, 1659-1672 (2004).

94. Yonkin, M., Clubine, K. \& O’Connor, K. Importance of energy efficiency to the water and wastewater sector. Clear Waters 38, 12-13 (2008).

95. Pheasant, R. \& Tait, S. INNERS literature review: An overview of energy used within the urban water cycle. Europe 2025, 2008-2035 (2010).

96. Elimelech, M.; Phillip, W. A., The future of seawater desalination: energy, technology, and the environment. Science 2011, 333, (6043), 712-717.

97. Fan, Y.; Li, H.; Miguez-Macho, G., Global patterns of groundwater table depth. Science 2013, 339, (6122), 940-943.

98. International Energy Agency (IEA), Energy Balances of OECD Countries 2012. OECD Publishing: Paris, 2012.

99. Edmonds, J., M. Wise, H. Pitcher, R. Richels, T. Wigley, and C. MacCracken. An Integrated Assessment of Climate Change and the Accelerated Introduction of Advanced Energy Technologies, Mitigation and Adaptation Strategies for Global Change, 1997, 1, 311-339 